\title{
The entanglement entropy of one-dimensional systems in continuous and homogenous space
}

\author{
Pasquale Calabrese, Mihail Mintchev, and Ettore Vicari \\ Dipartimento di Fisica dell'Università di Pisa and INFN, Pisa, Italy
}

\begin{abstract}
.
We introduce a systematic framework to calculate the bipartite entanglement entropy of a compact spatial subsystem in a one-dimensional quantum gas which can be mapped into a noninteracting fermion system. We show that when working with a finite number of particles $N$, the Rényi entanglement entropies grow as $\ln N$, with a prefactor that is given by the central charge. We apply this novel technique to the ground state and to excited states of periodic systems. We also consider systems with boundaries. We derive universal formulas for the leading behavior and for subleading corrections to the scaling. The universality of the results allows us to make predictions for the finite-size scaling forms of the corrections to the scaling.
\end{abstract}


The entanglement entropy of $1 D$ systems in continuous and homogenous space

\section{Introduction}

Entanglement is a fundamental phenomenon of quantum mechanics. Much theoretical work has focused on the entanglement properties of quantum many-body systems, showing their importance to characterize the many-body dynamics [1]. In particular, lots of studies have been devoted to quantify the nontrivial connections between different parts of an extended quantum system, by computing von Neumann or Rényi entanglement entropies of the reduced density matrix $\rho_{A}$ of a subsystem $A$. Rényi entanglement entropies are defined as

$$
S_{\alpha}=\frac{1}{1-\alpha} \ln \operatorname{Tr} \rho_{A}^{\alpha} .
$$

For $\alpha \rightarrow 1$ this definition gives the most commonly used von Neumann entropy $S_{1}=-\operatorname{tr} \rho_{A} \ln \rho_{A}$, while for $\alpha \rightarrow \infty$ is the logarithm of the largest eigenvalue of $\rho_{A}$ also known as single copy entanglement [2].

One of the most remarkable results is the universal behavior displayed by the entanglement entropy at $1 \mathrm{D}$ conformal quantum critical points (i.e. with dynamical critical exponent $z=1$ ), determined by the central charge [3] of the underlying conformal field theory (CFT) [4, 5, 6, 7]. For a partition of an infinite 1D system into a finite piece $A$ of length $\ell$ and the remainder, the Rényi entanglement entropies for $\ell$ much larger than the short-distance cutoff $a$ are

$$
S_{\alpha}=\frac{c}{6}\left(1+\frac{1}{\alpha}\right) \ln \frac{\ell}{a}+c_{\alpha},
$$

where $c$ is the central charge and $c_{\alpha}$ a non-universal constant. When $A$ is a finite interval of length $\ell$ in a finite periodic system of length $L$, CFT predicts the universal asymptotic scaling [6]

$$
S_{\alpha}=\frac{c}{6}\left(1+\frac{1}{\alpha}\right) \ln \left(\frac{L}{\pi a} \sin \frac{\pi \ell}{L}\right)+c_{\alpha},
$$

where, remarkably $c_{\alpha}$ is the same non-universal constant in Eq. 22). For future reference, it is also important to mention the result in a finite system of length $L$ with some boundary conditions at its ends and for an interval of length $\ell$ starting from one of the two boundaries [6, 8, 9]:

$$
S_{\alpha}=\frac{c}{12}\left(1+\frac{1}{\alpha}\right) \ln \left(\frac{2 L}{\pi a} \sin \frac{\pi \ell}{L}\right)+\frac{c_{\alpha}}{2}+\ln g,
$$

where again $c_{\alpha}$ is the same non-universal constant as above and $\ln g$ is the universal boundary entropy of Affleck and Ludwig [10]. All the Rényi entropies $S_{\alpha}$ are proper and equivalent measure of entanglement in a pure state [1, but the knowledge of $S_{\alpha}$ for different $\alpha$ characterizes the full spectrum of non-zero eigenvalues of $\rho_{A}$ (see e.g. [11) providing significantly more information on the entanglement than the solely knowledge of the von Neumann entropy.

The CFT results reviewed above have been confirmed in many spin chains and in 1D itinerant systems on the lattice (too many to be mentioned here, we remand the interested reader to the comprehensive reviews on the subject [1]). These studies have allowed a deeper understanding of the convergence and precision [12] of 1D simulation algorithms based on the so-called matrix product states [13. However, analogous results must also be valid for systems in continuous space, and therefore directly derivable in continuous models. Apart from the interest to describe trapped 
1D gases experimentally realized with cold atoms, the entanglement of continuous models is also instrumental to develop 1D tensor network algorithms for gases, as the one proposed in [14. Despite of this fundamental interest, almost no effort (with the exception of Refs. [15, 16] and the orbital partitioning in quantum Hall states [17) has been devoted to the spatial entanglement of gas models (that is distinguished from the particle partitioning [18]).

In a previous short communication [19] we introduced a systematic framework to tackle free fermion gases in any external conditions for an arbitrarily large number of particles. The most general result of this investigation was that, when dealing with a finite number of particles $N$, the $1 \mathrm{D}$ entanglement entropy grows like $\ln N$, with a prefactor that again is given by the central charge. In this formulation $N$ acts as an UV cutoff, representing a concrete alternative to the lattice. In this manuscript, we detail the calculations in Ref. [19] for homogenous 1D gases and we report a series of new results about the leading and subleading corrections to their scaling behavior. The degree of universality of these results allows to us to make novel predictions for spin chains on some universal functions describing the corrections to the scaling. The determination of these functions was left as open problem by previous lattice investigations 20, 21, 22,

\subsection{The model and its equivalence with others}

We consider a system of free spinless fermions in the continuum interval $[0, L]$. We work with a finite number of particles $N$. Therefore all the quantities and in particular entanglement entropies are finite since $N$ acts as a cutoff. Appropriate boundary conditions (BC) are imposed in order to have a discrete energy spectrum.

Apart the per se interest, spinless free fermions are also equivalent to other models of direct physical application. The 1D Bose gas with short-ranged repulsive interaction (i.e. the Lieb-Liniger model [23]) with Hamiltonian

$$
\mathcal{H}_{N}=-\sum_{j=1}^{N} \frac{\partial^{2}}{\partial x_{j}^{2}}+2 C \sum_{1 \leq j<l \leq N} \delta\left(x_{j}-x_{l}\right),
$$

in the limit of strong interaction $C \rightarrow \infty$ (i.e. impenetrable bosons, also known as Tonk-Girardeau gas) is exactly mapped to spinless fermions 24 and the entanglement entropy of a single interval in the two models do coincide, because the boson in an interval are functions only of the fermions in the same interval (this is not true anymore in the case of more disjoint intervals because of the presence of a bosonization string, analogously to spin-chain models [25, 26, 27]). The properties of the Lieb-Liniger model are described solely by the dimensionless parameter $\gamma=C L / N[23$, thus the Tonks-Girardeau limit describes the dilute model (i.e. $N / L \ll 1$ ) for any value of $C$.

Another important model mappable to free fermions is the spin- $1 / 2 \mathrm{XX}$ chain defined by the Hamiltonian

$$
H=-\sum_{l=0}^{L} \frac{1}{2}\left[\sigma_{l}^{x} \sigma_{l+1}^{x}+\sigma_{l}^{y} \sigma_{l+1}^{y}\right]-h \sigma_{l}^{z},
$$

where $\sigma_{l}^{x, y, z}$ are the Pauli matrices at site $l$. The Jordan-Wigner transformation

$$
c_{l}=\left(\prod_{m<l} \sigma_{m}^{z}\right) \frac{\sigma_{l}^{x}+i \sigma_{l}^{y}}{2},
$$


maps this model to the quadratic Hamiltonian of spinless fermions

$$
H=-\sum_{l=0}^{L} c_{l}^{\dagger} c_{l+1}+c_{l+1}^{\dagger} c_{l}+2 h\left(c_{l}^{\dagger} c_{l}-\frac{1}{2}\right) \text {. }
$$

Here $h$ represents the chemical potential for the spinless fermions $c_{l}$, which satisfy canonical anti-commutation relations $\left\{c_{l}, c_{m}^{\dagger}\right\}=\delta_{l, m}$. The Hamiltonian (8) is diagonal in momentum space and for $|h|<1$ the ground-state is a Fermi sea with filling

$$
\nu=\frac{\arccos |h|}{\pi} .
$$

Only for $|h|<1$ we are dealing with a gapless theory.

The continuum limit of the Hamiltonian (8) is then the system of free-fermions we are considering in this paper and so all the universal properties that do not depend on lattice regularization can be obtained from the continuum model. At this point, it is worth discussing how to obtain the continuum limit in some details. The lattice model is formed by $L_{\text {lat }}$ sites separated by the lattice spacing $a$ (usually set to 1 in all lattice studies). $N$ particles populate the chain with filling $\nu=N / L_{\text {lat }}$ and we are interested in the entanglement entropy of $\ell_{\text {lat }}$ sites. The continuum limit is a system of $N$ free fermions in a box of length $L$ and is obtained by sending $a \rightarrow 0, \nu \rightarrow 0$ keeping fixed $\nu \ell_{\text {lat }}$ equal to $\ell N / L$ in the continuum, where $L=a L_{\text {lat }}$ and $\ell=a \ell_{\text {lat }}$. This allows us to use the CFT results to predict a priori some of the results we are going to derive. In Ref. 28, Eq. 22) has been derived for the XX chain, obtaining

$$
S_{\alpha}^{X X}=\frac{c}{6}\left(1+\frac{1}{\alpha}\right) \ln \left(\frac{\ell_{\mathrm{lat}}}{a} 2 \sin \pi \nu\right)+E_{\alpha},
$$

where also the non-universal constant is determined as

$E_{\alpha}=\left(1+\frac{1}{\alpha}\right) \int_{0}^{\infty} \frac{d t}{t}\left[\frac{1}{1-\alpha^{-2}}\left(\frac{1}{\alpha \sinh t / \alpha}-\frac{1}{\sinh t}\right) \frac{1}{\sinh t}-\frac{e^{-2 t}}{6}\right]$.

Combining this exact result with the CFT prediction in Eq. 33, we get the asymptotic scaling behavior of the entanglement entropies in finite $\mathrm{XX}$ chain

$$
S_{\alpha}^{X X}=\frac{c}{6}\left(1+\frac{1}{\alpha}\right) \ln \left[\frac{L_{\mathrm{lat}}}{\pi a} \sin \left(\frac{\pi \ell_{\mathrm{lat}}}{L_{\mathrm{lat}}}\right) 2 \sin \pi \nu\right]+E_{\alpha} .
$$

Taking now the continuum limit $a \rightarrow 0, \nu \rightarrow 0$ as explained above, we arrive at the prediction

$S_{\alpha}^{\mathrm{cont}} \equiv \lim _{a \rightarrow 0}\left[S_{\alpha}^{X X}-\frac{1}{6}\left(1+\frac{1}{\alpha}\right) \ln (1 / a)\right]=\frac{1}{6}\left(1+\frac{1}{\alpha}\right) \ln \left(2 N \sin \pi \frac{\ell}{L}\right)+E_{\alpha}$,

where the subtraction of the term proportional to $\ln a$ comes from the normalization of the reduced density matrix.

Finally we quote the 1D Bose-Hubbard model described by the Hamiltonian

$$
H_{\mathrm{BH}}=\frac{J}{2} \sum_{i}\left(b_{i+1}-b_{i}\right)^{\dagger}\left(b_{i+1}-b_{i}\right)+\frac{U}{2} \sum_{i} n_{i}\left(n_{i}-1\right),
$$

where $b_{i}$ are bosonic operators and $n_{i} \equiv b_{i}^{\dagger} b_{i}$ is the particle density operator. The hard-core limit $U \rightarrow \infty$ of the Bose-Hubbard model implies that the particle number $n_{i}$ per site is restricted to the values $n_{i}=0,1$, and so in this limit can be exactly mapped into a lattice model of spinless fermions. Clearly the continuum limit of the Bose-Hubbard model is nothing but the Lieb-Liniger gas introduced above. 
The entanglement entropy of $1 D$ systems in continuous and homogenous space

\section{The method}

We consider a system of $N$ non-interacting spinless fermions with discrete one-particle energy spectrum, such as a finite system or one confined by a proper external potential. The many body wave functions $\Psi\left(x_{1}, \ldots, x_{N}\right)$ can be written in terms of the oneparticle eigenstates as a Slater determinant

$$
\Psi\left(x_{1}, \ldots, x_{N}\right)=\frac{1}{\sqrt{N !}} \operatorname{det}\left[\phi_{k}\left(x_{n}\right)\right],
$$

where the normalized wave functions $\phi_{k}(x)$ represent the occupied single-particle energy levels. The ground state is obtained by filling the $N$ levels with lowest energies. Thus, the ground-state two-point correlator is

$$
C(x, y) \equiv\left\langle c^{\dagger}(x) c(y)\right\rangle=\sum_{k=1}^{N} \phi_{k}^{*}(x) \phi_{k}(y)
$$

where $c(x)$ is the fermionic annihilation operator and the one-particle eigenfunctions $\phi_{k}(x)$ are intended to be ordered according to their energies. The reduced density matrix of a subsystem $A$ extending from $x_{1}$ to $x_{2}$ can be written as

$$
\rho_{A} \propto \exp \left(-\int_{x_{1}}^{x_{2}} d y_{1} d y_{2} c^{\dagger}\left(y_{1}\right) \mathcal{H}\left(y_{1}, y_{2}\right) c\left(y_{2}\right)\right),
$$

where $\mathcal{H}=\ln [(1-C) / C]$ and the normalization constant is fixed requiring $\operatorname{Tr} \rho_{A}=1$. This equation can be straightforwardly seen as the continuum limit of the formula for lattice free fermions 29, 30, but can also be obtained following the standard derivation in Ref. [29] in path integral formalism. In the passage from lattice to continuum, the normalization factor in (17) depends explicitly on the lattice spacing $a$ and it is responsible for the subtraction of the term proportional to $\ln a$ in Eq. (13).

We want to compute the bipartite Renyi entanglement entropies defined as in Eq. (1) of the space interval $A$ in this fermion gas. For this purpose, we introduce the Fredholm determinan 7

$$
D_{A}(\lambda)=\operatorname{det}\left[\lambda \delta_{A}(x, y)-C_{A}(x, y)\right],
$$

where $C_{A}(x, y)$ is the restriction of $C(x, y)$ to the part at hand from $x_{1}$ to $x_{2}$ that can be written as $C_{A}(x, y)=\theta\left(x-x_{1}\right) \theta\left(x_{2}-x\right) C(x, y) \theta\left(y-x_{1}\right) \theta\left(x_{2}-y\right)$ (or in matrix form $C_{A}=P_{A} C P_{A}$, where $P_{A}$ is the projector on the interval $A$ ). The same definition holds for $\delta_{A}(x, y)=P_{A} \delta(x-y) P_{A}$. Following the ideas for the lattice model [28], $D_{A}(\lambda)$ can be introduced in such a way that it is a polynomial in $\lambda$ having as zeros the $N$ eigenvalues of $C_{A}$. The Gaussian form of $\rho_{A}$ in Eq. 17) allows us to exploit the relation between the eigenvalues of $\rho_{A}$ and $C_{A}$ to write

$$
S_{\alpha} \equiv \frac{\ln \operatorname{Tr} \rho_{A}^{\alpha}}{1-\alpha}=\oint \frac{d \lambda}{2 \pi i} e_{\alpha}(\lambda) \frac{d \ln D_{A}(\lambda)}{d \lambda},
$$

¥ A Fredholm determinant is the extension of the standard determinant to continuous matrices. Its simplest operative definition is through the generalization to continuous kernels $K(x, y)$ of the standard identity for determinants of a finite matrix $\mathbb{M}$

$\ln \operatorname{det}[\lambda \mathbb{I}-\mathbb{M}]=-\sum_{k=1}^{\infty} \frac{\operatorname{Tr} \mathbb{M}^{k}}{k \lambda^{k}} \Longrightarrow \ln \operatorname{det}[\lambda \delta(x-y)-K(x, y)]=-\sum_{k=1}^{\infty} \frac{\operatorname{Tr} K^{k}}{k \lambda^{k}}$,

where the traces are simply

$$
\operatorname{Tr} K^{n}=\int d x_{1} d x_{2} \ldots d x_{n} K\left(x_{1}, x_{2}\right) K\left(x_{2}, x_{3}\right) \ldots K\left(x_{n-1}, x_{n}\right) K\left(x_{n}, x_{1}\right) .
$$


where the integration contour encircles the segment $[0,1]$, and

$$
e_{\alpha}(\lambda)=\frac{1}{1-\alpha} \ln \left[\lambda^{\alpha}+(1-\lambda)^{\alpha}\right] .
$$

For $\alpha \rightarrow 1, e_{1}(\lambda)=-x \ln x-(1-x) \ln (1-x)$ and Eq. (21) reproduces the von Neumann definition. The integral representation 21 has been already derived and used in the context of discrete chain models [28, thus involving the determinant of a standard matrix with the lattice sites as indices.

The Fredholm determinant is turned into a standard one by introducing the $N \times N$ reduced overlap matrix $\mathbb{A}$ (also considered in Ref. [15]) with elements

$$
\mathbb{A}_{n m}=\int_{x_{1}}^{x_{2}} d z \phi_{n}^{*}(z) \phi_{m}(z), \quad n, m=1, \ldots, N
$$

such that

$$
\begin{aligned}
\operatorname{Tr} C_{A}^{k} & =\int_{x_{1}}^{x_{2}}\left(\prod_{j=1}^{k} d y_{j}\right) C_{A}\left(y_{1}, y_{2}\right) \ldots C_{A}\left(y_{k-1}, y_{k}\right) C_{A}\left(y_{k}, y_{1}\right)= \\
& =\int_{x_{1}}^{x_{2}}\left(\prod_{j=1}^{k} d y_{j}\right) \sum_{n_{1}=1}^{N} \phi_{n_{1}}^{*}\left(y_{1}\right) \phi_{n_{1}}\left(y_{2}\right) \ldots \sum_{n_{k}=1}^{N} \phi_{n_{k}}^{*}\left(y_{k}\right) \phi_{n_{k}}\left(y_{1}\right)= \\
& =\sum_{n_{1}=1}^{N} \ldots \sum_{n_{k}=1}^{N} \int_{x_{1}}^{x_{2}} d y_{1} \phi_{n_{1}}^{*}\left(y_{1}\right) \phi_{n_{k}}\left(y_{1}\right) \ldots \int_{x_{1}}^{x_{2}} d y_{k} \phi_{n_{k}}^{*}\left(y_{k}\right) \phi_{n_{k-1}}\left(y_{k}\right)=\operatorname{Tr} \mathbb{A}^{k},
\end{aligned}
$$

where from the first to the second line we use Eq. (16). Thus

$$
\ln D_{A}(\lambda)=-\sum_{k=1}^{\infty} \frac{\operatorname{Tr} C_{A}^{k}}{k \lambda^{k}}=-\sum_{k=1}^{\infty} \frac{\operatorname{Tr} \mathbb{A}^{k}}{k \lambda^{k}}=\ln \operatorname{det}[\lambda \mathbb{I}-\mathbb{A}]=\sum_{m=1}^{N} \ln \left(\lambda-a_{m}\right),
$$

where we use twice Eq. 18 and we denote with $a_{m}$ the eigenvalues of $\mathbb{A}$. Inserting it into the integral 21, we obtain

$$
S_{\alpha}\left(x_{1}, x_{2}\right)=\oint \frac{d \lambda}{2 \pi i} \sum_{m=1}^{N} \frac{e_{\alpha}(\lambda)}{\lambda-a_{m}}=\sum_{m=1}^{N} e_{\alpha}\left(a_{m}\right),
$$

as a consequence of the residue theorem.

The matrix $\mathbb{A}$ is easily obtained for any non-interacting model from the oneparticle wave functions, as the definition 23 shows. Calculating the entanglement entropies is then reduced to an $N \times N$ eigenvalue problem that can be easily solved numerically and in some instances even analytically, as we are going to show.

\section{The ground-state of systems with periodic boundary conditions}

In a system of length $L$ with periodic boundary conditions (BC), the normalized oneparticle wave-functions are plane waves with integer wave numbers

$$
\phi_{k}(x)=\frac{e^{2 \pi i k x / L}}{\sqrt{L}}, \quad k \in \mathbb{Z},
$$

and energy $E_{k}=2 \pi^{2} k^{2} / L^{2}$. For some physical problems, one has to impose antiperiodic $\mathrm{BC}$ for the fermion degrees of freedom (the appropriate $\mathrm{BC}$ can also depends on the parity of $N$ ) and so the momentum is quantized in terms of semi-integer wave numbers. However, as long as we are interested in the entanglement entropy of a 
single interval, this does not change the final results because, as we shall see soon, the elements of the matrix $\mathbb{A}$ depends only on the difference between momenta that are always integer. Different results would be instead obtained for the entanglement of two disjoint intervals, similarly to what happens in CFT [31, 32] and lattice models [25, 26, 27.

The element of the overlap matrix between two one-particle eigenstates with wave number $k_{1}$ and $k_{2}$ is

$$
\begin{aligned}
\mathbb{A}_{k_{1} k_{2}} & =\frac{1}{L} \int_{x_{1}}^{x_{2}} d z e^{-2 \pi i k_{1} x / L} e^{2 \pi i k_{2} x / L} \\
& =e^{\pi i\left(k_{2}-k_{1}\right)\left(x_{1}+x_{2}\right) / L} \frac{\sin \pi\left(k_{1}-k_{2}\right)\left(x_{2}-x_{1}\right) / L}{\pi\left(k_{1}-k_{2}\right)} .
\end{aligned}
$$

The elements of the matrix $\mathbb{A}$ are not invariant under translation because of the explicit dependence on $x_{1}+x_{2}$ of the phase factor. However, the eigenvalues of $\mathbb{A}$ do not depend on this phase factor (but the eigenvectors do) and so also the entanglement entropies are translational invariant, as they must be. Indeed, in the determinant of $\lambda \mathbb{I}-A$, for each column we can bring out of the determinant the factor $e^{-\pi i k_{1}\left(x_{1}+x_{2}\right) / L}$ and for each row $e^{\pi i k_{2}\left(x_{1}+x_{2}\right) / L}$. Since $k_{1}$ and $k_{2}$ run on the same set of integers, the product of all these phases is 1 , regardless of the values of the $k \mathrm{~s}$. In the following we use this freedom to fix the phase factor to 1 and denote $\ell=x_{2}-x_{1}$.

The ground state of a fermion gas with $N$ particles is obtained by filling the $N$ $k$-modes with lowest energies. In the case of odd $N$, this amounts to fill symmetrically the $N$ states with $|k| \leq(N-1) / 2$ (the zero mode is clearly included). For even $N$, there are two degenerate states obtained from the $N-1$ ground state by filling the first available state either on the right or on the left. This small difference between odd and even terms does not play any role because the elements of the matrix $\mathbb{A}$ in Eq. (28) depend only on the differences between $k_{1}$ and $k_{2}$. Thus we can just start counting modes from the lowest $k$ one-particle occupied state and the resulting matrix $\mathbb{A}$ for a segment of length $\ell=x_{2}-x_{1}$ is from Eq. 28

$$
\mathbb{A}_{n m}=\frac{\sin \pi(n-m) \ell / L}{\pi(n-m)}, \quad n, m=1, \ldots, N .
$$

By inserting the $N$ eigenvalues of $\mathbb{A}$ into Eq. (26), we obtain the entanglement entropy in a system of $N$ particles. This is very easily done numerically as in Fig. 1 .

\subsection{The leading behavior of the entanglement entropies}

We can also compute analytically the large $N$ behavior of the entanglement entropies. Indeed we can write $\ln D_{A}=\ln \operatorname{det} \mathbb{G}$, where $\mathbb{G} \equiv \lambda \mathbb{I}-\mathbb{A}$ is an $N \times N$ Toeplitz matrix. We can then use the Fisher-Hartwig conjecture [33] to rigorously $[$ calculate the large $N$ behavior of $S_{\alpha}$. However, going through all the technical complications of the Fisher-Hartwig conjecture is not needed, because we can exploit the results obtained for the very similar matrices of lattice free fermions on the infinite line. In fact, for lattice models in the thermodynamic limit at fixed filling $\nu=N / L_{\text {lat }}$, it has been

found that the entanglement entropies of $\ell_{\text {lat }}$ consecutive sites is given by Eq. 21)

$\S$ Despite its name, the Fisher-Hartwig conjecture has been rigorously proven for the case at hand (see e.g. Ref. [34]). 


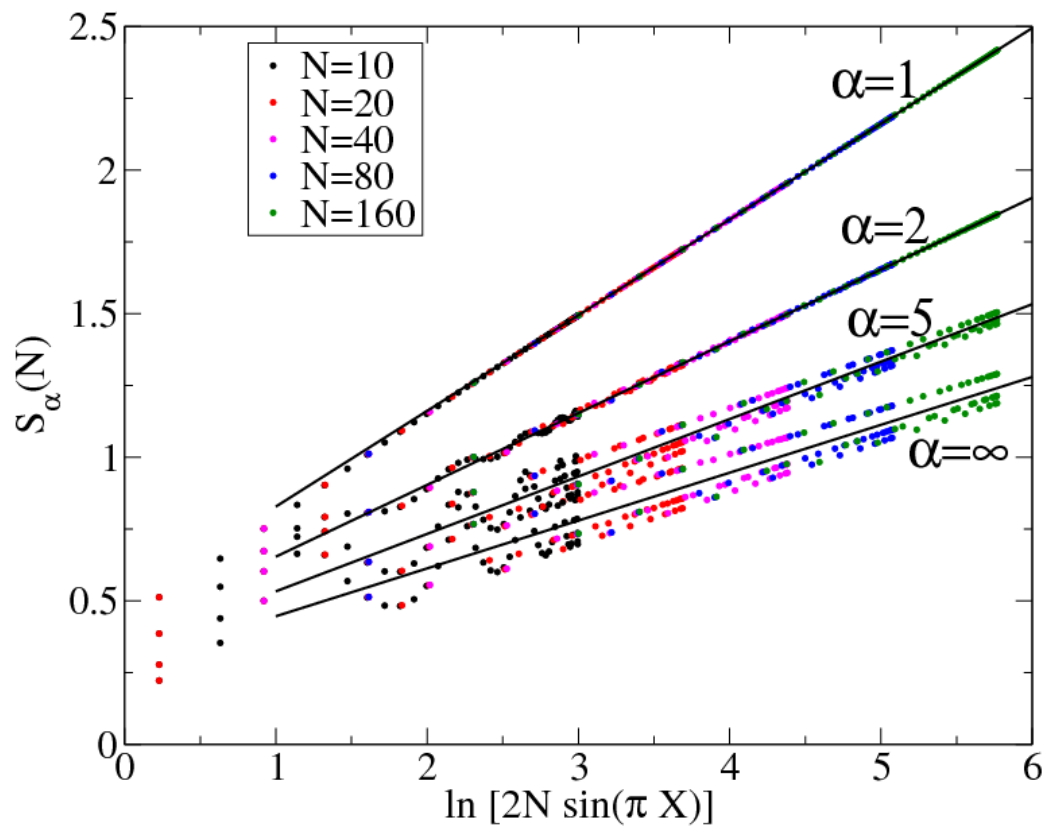

Figure 1. $S_{\alpha}(N)$ for $\alpha=1,2,5, \infty$ (from top to bottom) as function of $\ln [2 N \sin (\pi X)]$ with $X=\ell / L$ calculated numerically for several values of $N=10,20,40,80,160$. The straight lines are the asymptotic predictions in Eq. 31. The convergence is clear, although for $\alpha>1$ non-monotonic corrections to the asymptotic behavior are present.

where $D_{A}(\lambda)$ is a standard $\ell_{\text {lat }} \times \ell_{\text {lat }}$ determinant with the correlation matrix $\mathbf{C}$ given by 30,35$]$

$$
\mathbb{C}_{n m}^{\mathrm{lat}}=\frac{\sin \pi \nu(n-m)}{\pi(n-m)}, \quad n, m=1, \ldots, \ell_{\mathrm{lat}} .
$$

It is evident that this matrix $\mathbb{C}_{n m}^{\text {lat }}$ is the same as $\mathbb{A}$ in Eq. 29 identifying $\nu$ with $\ell / L$. However, this is only a mathematical coincidence and it will most probably not be true for interacting systems. Indeed, in Eq. $\sqrt{29}$ we have a finite system and the indices are related to occupation modes of the $N$ particles in the full system. On the other hand, in Eq. (30) we have an infinite lattice with filling $\nu$ and the indices refer to the lattice sites of the subsystem. Having established this equivalence between the two matrices, we can use the exact calculations in Ref. 28 (see also 21]), replace $\nu$ with $\ell / L$, and obtain the asymptotic behavior of the desired entanglement entropies as

$$
S_{\alpha}=\frac{1}{6}\left(1+\frac{1}{\alpha}\right) \ln \left(2 N \sin \pi \frac{\ell}{L}\right)+E_{\alpha}+o\left(N^{0}\right) .
$$

Eq. (31) agrees with the CFT prediction for finite systems with periodic BC in Eq. (3) and represents an explicit analytic confirmation of this CFT result. It coincides with the scaling prediction in Eq. 13 from the lattice model, but here it has been derived from first principles.

Notice that we cannot recover the infinite volume limit from Eq. 29 because this limit must be taken at fixed ratio $N / L$. If we naively take $L \rightarrow \infty$ in Eq. 29) 


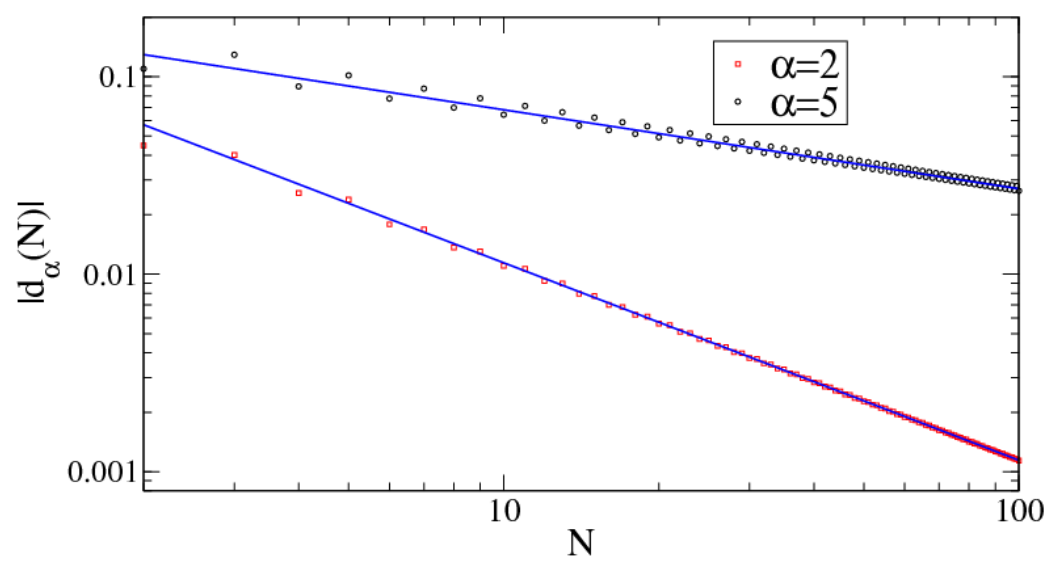

Figure 2. Leading asymptotic correction to the Rényi entanglement entropies of half system $\ell=L / 2$ for $N$ up to 100 and $\alpha=2,5$. Straight lines correspond to the prediction $(33)$ that agrees with numerical data. Note that also subleading corrections oscillate and can be described by Eq. (34.

we get a meaningless result, reflecting the non-commutation of the limits. Oppositely, after computing the determinant as in Eq. (31), the infinite volume limit exists at finite density $N / L$.

Fig. 1 shows a comparison with exact finite- $N$ calculations, for $\alpha=1,2,5, \infty$. It is evident that (especially for large $\alpha$ ) the data are affected by finite $N$ corrections that are exactly calculated in the next subsection.

\subsection{Corrections to the asymptotic behavior and universal FSS in finite chains}

The above correspondence, between the determinants giving the entanglement entropies of the continuos system and the ones for spin chains, permits a quantitative description of the scaling correction to the leading behavior in Eq. (31) by exploiting the results based on the generalized Fisher-Hartwig conjecture [36] in Refs. 20, 21. We introduce the differences between the entanglement at finite $N$ and the asymptotic values $S_{\alpha}^{\text {asy }}(N)$ in Eq. 31 as

$$
d_{\alpha}(N) \equiv S_{\alpha}(N)-S_{\alpha}^{\text {asy }}(N) .
$$

We can again use the spin-chain results of Refs. 20, 21, where a quantity analogous to $d_{\alpha}(N)$ was calculated at the leading order. Using these results and replacing $\nu$ with $\ell / L$ (in [28, 20, 21] $k_{F}=\pi \nu$ was used, but here we prefer to use $\nu$ to avoid confusion with the Fermi momentum in the continuous system), we obtain that the leading correction term is given by

$d_{\alpha}(N)=\frac{2 \cos (2 \pi \ell N / L)}{1-\alpha}(2 N \sin \pi \ell / L)^{-2 / \alpha}\left[\frac{\Gamma\left(\frac{1}{2}+\frac{1}{2 \alpha}\right)}{\Gamma\left(\frac{1}{2}-\frac{1}{2 \alpha}\right)}\right]^{2}+O\left(N^{-\min [4 / \alpha, 2]}\right)$.

A check of the correctness of this expression is reported in Fig. 2, where we report the absolute value of $d_{\alpha}(N)$ for the half system entanglement (i.e. $\ell / L=1 / 2$ ) for $N$ up to 100. The power law behavior is evident and the straight lines are given by Eq. (33) without any adjustable parameter. These corrections of the form $N^{-2 / \alpha}$ correspond to the $\ell^{-2 / \alpha}$ corrections found within conformal field theory [37, that have already been 
generalized to other situations, such as massive field theories [38, confined systems 39], disordered models [40, and have been carefully checked numerically in many different models [20, 41, 22].

Subleading corrections to the scaling are visible in Fig. 2, and for large values of $\alpha$ they have a sizable effect. These can be exactly calculated adapting the results of Ref. [21] (based on generalized Fisher-Hartwig [36] and random matrix theory [42]) and by replacing $\nu$ with $\ell / L$. The full result for $d_{\alpha}(\ell)$ up to order $N^{-3}$ can be cast in the form

$$
\begin{aligned}
d_{\alpha}(N)= & \frac{2}{\alpha-1} \sum_{p, q=1}^{\infty}(-1)^{p} L_{N}^{-\frac{2 p(2 q-1)}{\alpha}}\left(Q_{q}\right)^{p}\left[\frac{\cos (2 p \pi N \ell / L)}{p}+\frac{A_{q} \sin (2 p \pi N \ell / L)}{L_{N}}\right. \\
& \left.+\frac{\left[B_{p, q} e^{2 i p \pi N \ell / L}+\text { h.c. }\right]}{L_{N}^{2}}\right] \\
& +\frac{1}{L_{N}^{2}} \frac{\alpha+1}{285 \alpha^{3}}\left(15\left(3 \alpha^{2}-7\right)+\left(49-\alpha^{2}\right) \sin ^{2}(\pi \ell / L)\right)+O\left(L_{N}^{-3}\right),
\end{aligned}
$$

where

$$
\begin{aligned}
L_{N}= & 2 N \sin (\pi \ell / L), \\
A_{q}= & {\left[1+3\left(\frac{2 q-1}{\alpha}\right)^{2}\right] \cos (\pi \ell / L), } \\
Q_{q}= & {\left[\frac{\left.\Gamma\left(\frac{1}{2}+\frac{2 q-1}{2 \alpha}\right)\right]^{2}}{\Gamma\left(\frac{1}{2}-\frac{2 q-1}{2 \alpha}\right)},\right.} \\
B_{p, q}= & \frac{2 q-1}{6 \alpha}\left[\left(5+7 \frac{(2 q-1)^{2}}{\alpha^{2}}\right) \sin ^{2}(\pi \ell / L)-15\left(\frac{(2 q-1)^{2}}{\alpha^{2}}+1\right)\right] \\
& -\frac{p}{4}\left[\left(1+3 \frac{(2 q-1)^{2}}{\alpha^{2}}\right) \cos (\pi \ell / L)\right]^{2} .
\end{aligned}
$$

As for the spin chain, while Eq. (34) provides an infinite number of contributions, for a given fixed value of $\alpha$ only a finite number of them will be smaller than the leading neglected term, which is always of order $O\left(N^{-3}\right)$. To be specific, in the cases $\alpha=2,3,10 \mathrm{Eq}$. (34) gives the leading 4,8,46 terms in the asymptotic expansion of $d_{\alpha}(N)$ and hence the leading 6,10,48 terms in the expansion of $S_{\alpha}(\ell)$. We do not report here all the terms which contribute for specific values of $\alpha$ that can be obtained from a simple adaptation of the results above or from Ref. 21.

A remarkable exact result that we obtain from the previous analysis is the universal finite size scaling (FSS) form for finite XX spin chains with periodic BC. Indeed, as explained in section 1.1. the above result represents the continuum limit of the XX spin chain in a finite volume. For these spin chains, a universal FSS form has been observed in Ref. [20]. The quantity considered for the spin chain in Ref. [20] is

$F_{\alpha}(X)=\frac{d_{\alpha}\left(\ell_{\mathrm{lat}}, L_{\mathrm{lat}}\right)}{\frac{2}{1-\alpha}\left(\frac{L_{\mathrm{lat}}}{\pi} \sin (\pi X) 2 \sin \pi \nu\right)^{-2 / \alpha}\left[\frac{\Gamma\left(\frac{1}{2}+\frac{1}{2 \alpha}\right)}{\Gamma\left(\frac{1}{2}-\frac{1}{2 \alpha}\right)}\right]^{2}}, \quad$ with $X=\ell_{\mathrm{lat}} / L_{\mathrm{lat}}$.

In Ref. 20] chains with an odd number of spins $L_{\text {lat }}$ have been considered. Fig. 3 shows these results for $\alpha=2$ and magnetic field $h=0$. The figure shows a perfect data collapse and somehow the correctness of the FSS ansatz. Already in Ref. [20, it was observed that the scaling function is perfectly described by $F_{2}(X)= \pm \cos (\pi X)$, 


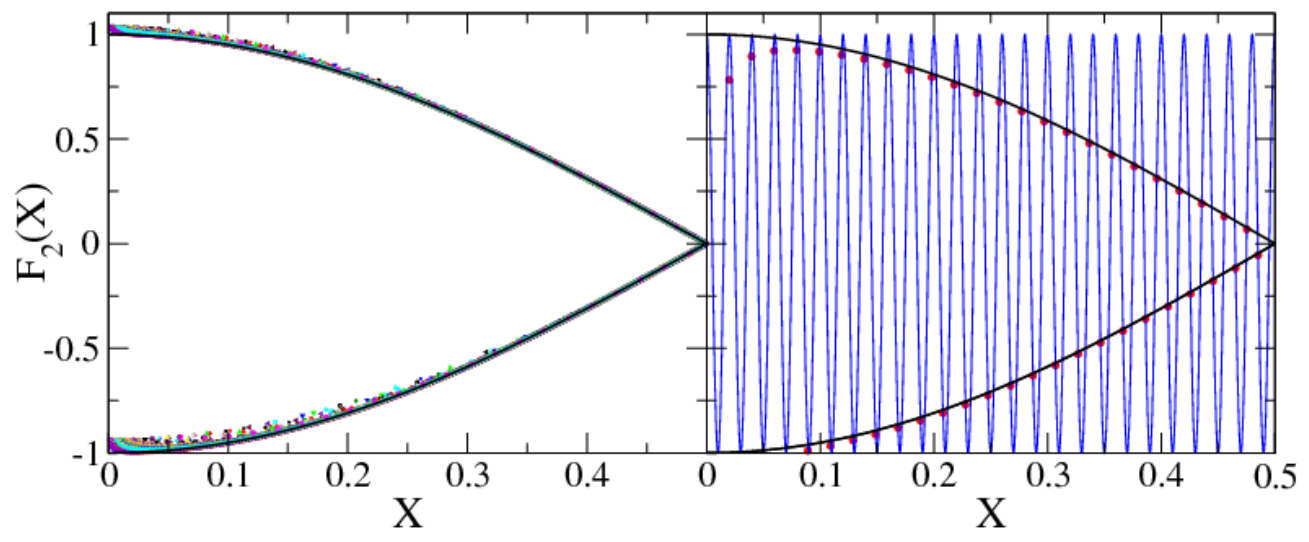

Figure 3. Universal function for the correction to the scaling of the XX spin chain (lattice free fermion) $F_{\alpha}(X)$ in Eq. (39) at zero magnetic field. Left: Numerical results for spin chains with $\alpha=2$, an odd number of spins $17 \leq L \leq 4623$, and all possible values of $\ell_{\text {lat }}<L_{\text {lat }} / 2$. Data show perfect data collapse to the function $F_{2}(X)= \pm \cos \pi X$. Right: numerical results for $L_{\text {lat }}=101$ against our prediction $F_{2}(X)=\cos \left(2 \pi \nu \ell_{\text {lat }}\right)$, showing graphically that the curve in the right panel is just the envelope obtained by sampling the oscillating function at integer values of $\ell_{\text {lat }}$ (see the text for details).

as evident result from the figure (where it is impossible to distinguish the data from the conjecture). However, this is in apparent contradiction with our result, suggesting that a FSS form does not exist and the quantity $F_{\alpha}(X)$ in Eq. (39) should instead be just a function of $\ell_{\text {lat }}$ and in particular

$$
F_{\alpha}\left(\ell_{\text {lat }}\right)=\cos \left(2 \pi \nu \ell_{\text {lat }}\right),
$$

where we changed the variable of $F_{\alpha}$ from $X$ to $\ell_{\text {lat }}$. To elucidate what is happening in the right panel of Fig. 3 we report the numerical data at $L_{\text {lat }}=101$ in zero magnetic field against our new prediction Eq. 40).

Being $L_{\text {lat }}$ odd, the ground state is not exactly at half-filling but at $\nu=$ $\left(L_{\text {lat }}-1\right) / 2 L_{\text {lat }}$. In Fig. 3 (right panel) we can observe that the prediction (40), i.e. the strongly oscillating curve, agrees with the numerical data, apart some subleading corrections to the scaling. The $\pm \cos (\pi X)$ form (also shown in the right panel) is nothing but the sampling of the curve at the integer values of $\ell_{\text {lat }}$. Indeed from basic trigonometry we have

$$
\begin{aligned}
\cos \left(2 \pi \nu \ell_{\text {lat }}\right) & =\cos \left(\pi \frac{L_{\text {lat }}-1}{L_{\text {lat }}} \ell_{\text {lat }}\right)=\cos \left(\pi \ell_{\text {lat }}-\pi \frac{\ell_{\text {lat }}}{L_{\text {lat }}}\right) \\
& =\cos \pi \ell_{\text {lat }} \cos \pi \frac{\ell_{\text {lat }}}{L_{\text {lat }}}+\sin \pi \ell_{\text {lat }} \sin \pi \frac{\ell_{\text {lat }}}{L_{\text {lat }}}=(-1)^{\ell_{\text {lat }}} \cos \pi X,
\end{aligned}
$$

where we used that $\ell_{\text {lat }}$ is an integer. The final expression is exactly the phenomenological result conjectured in Ref. [20] that we then prove and generalize here. Indeed for even chains, always in zero field, Ref. 20] proposed phenomenologically $F_{\alpha}(X)= \pm 1$, which corresponds to $\cos \left(2 \pi \nu \ell_{\text {lat }}\right)$ i.e., for $\nu=1 / 2$, to $(-1)^{\ell_{\text {lat }}}$. We checked Eq. 40 against other spin-chain results at different filling, always finding agreement. Leading corrections to the scaling having a structure similar to Eq. 40 have been conjectured in Ref. [43] for spin chains with open boundary conditions. 


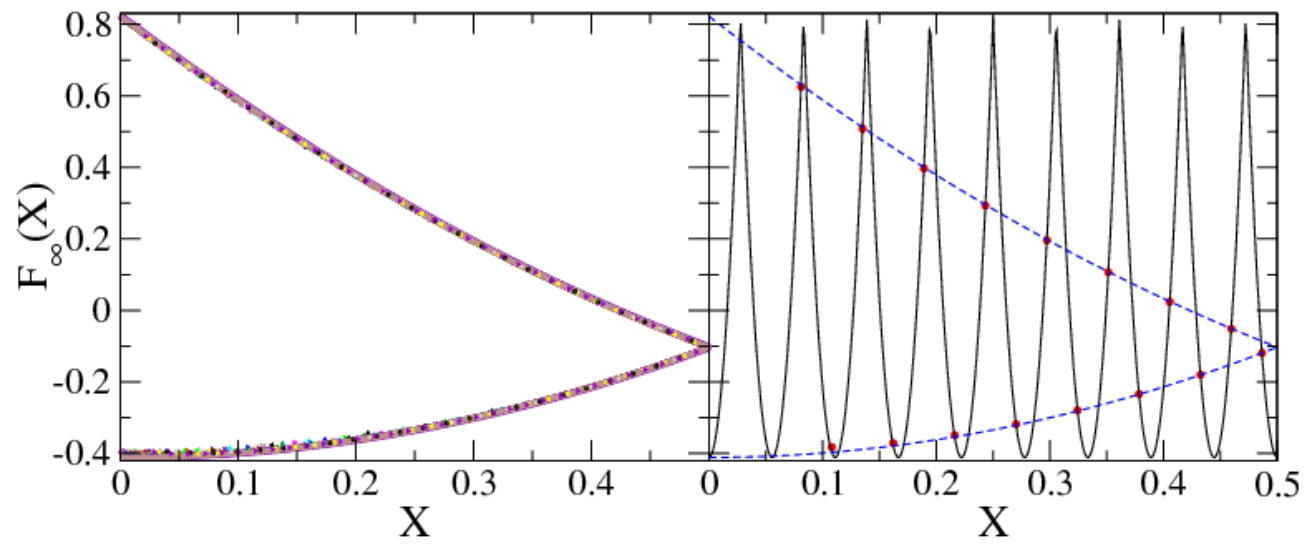

Figure 4. Universal function $F_{\infty}(X)$ for the correction to the scaling of the single copy entanglement in the XX spin chain at zero-magnetic field. Left: Numerical results for spin chains with an odd number of spins $17 \leq L \leq 4623$ and all possible values of $\ell_{\text {lat }}<L_{\text {lat }} / 2$. Data shows perfect data collapse to the function $F_{\infty}(X)$ in Eq. (45) shown as a continuous line, indistinguishable from data point. Right: numerical results for $L_{\text {lat }}=37$ against our prediction $F_{\infty}(X)$ in Eq. 44. It is graphically evident that the curve on the left is the envelope obtained by sampling the oscillating functions at integer values of $\ell_{\text {lat }}$.

3.2.1. The single copy entanglement: $\alpha \rightarrow \infty$. For $\alpha \rightarrow \infty$, the Rényi entanglement entropy gives the logarithm of the maximum eigenvalue of $\rho_{A}$ also known as single copy entanglement 2]. It is not possible to obtain the result at $\alpha \rightarrow \infty$ from the general form in Eq. (34) because all the corrections of the form $N^{-2 / \alpha}$ resum. Again instead of re-doing all the calculation to resum these terms, we exploit the correspondence with the infinite spin chain, and simply obtain the final result from Ref. 21] substituting $\nu$ with $\ell / L$. After straightforward algebra we obtain

$d_{\infty}(N)=\frac{1}{4 \ln [b N \sin (\pi \ell / N)]}\left[\operatorname{Li}_{2}\left(-e^{i 2 \pi \ell N / L}\right)+\operatorname{Li}_{2}\left(-e^{-i 2 \pi \ell N / L}\right)\right]$,

with $b=\exp (-\Psi(1 / 2)) \approx 7.12429$. We have checked these results against exact numerical computation that we do not report here.

It is interesting also in this case to explore the consequences of this result for finite spin chains, on the same lines as above for the finite $\alpha$ results. In Ref. 20, for chains with an odd number of spins, it has been shown that the data for several choices of $L_{\text {lat }}$ and $\ell_{\text {lat }}$ collapse on a single master curve if plotted as

$F_{\infty}(X)=\frac{d_{\infty}\left(\ell_{\text {lat }}, L_{\text {lat }}\right)}{\left[\ln \left(2 b \frac{L_{\text {lat }}}{\pi} \sin (\pi X) \sin (\pi \nu)\right)\right]^{-1}}, \quad$ with $X=\ell_{\text {lat }} / L_{\text {lat }}$.

Numerical data showing this collapse (analogous to those in Ref. 20]) are reported in Fig. 4 (left) for zero magnetic field and odd $L_{\text {lat }}$. Oppositely to the case for finite $\alpha$, the shape of this curve was too complicated to be guessed in Ref. 20. As before, assuming universality in the FSS towards the continuum limit, we predict

$$
F_{\infty}\left(\ell_{\text {lat }}\right)=\frac{1}{4}\left[\operatorname{Li}_{2}\left(-e^{i 2 \pi \nu \ell_{\text {at }}}\right)+\operatorname{Li}_{2}\left(-e^{-i 2 \pi \nu \ell_{\text {lat }}}\right)\right] .
$$

For a small chain with $L_{\mathrm{lat}}=37$, the above curve is reported in the right panel of Fig. 4 and it shows high frequency oscillations, but perfectly coincides with the exact lattice 

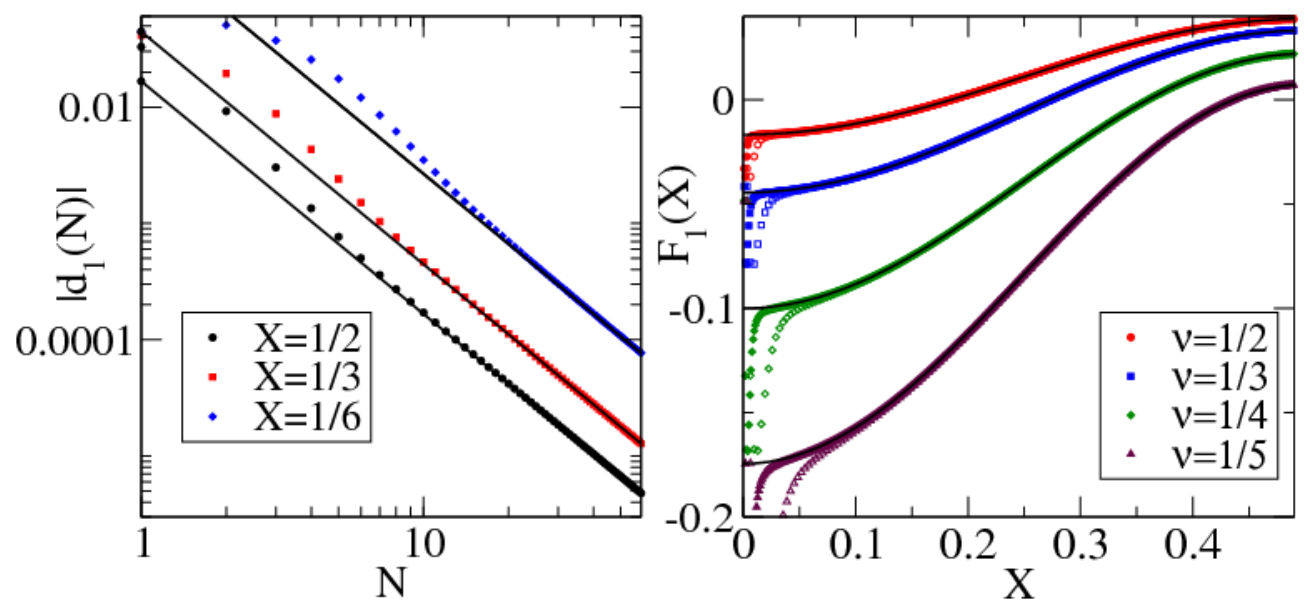

Figure 5. Left: Leading correction to the scaling for the von Neumann entanglement entropy for the free-fermion gas for several length of the subinterval $\ell=X L$ as function of $N$ up to 60 . The straight lines are the asymptotic prediction in Eq. 46). Right: Universal function $F_{1}(X)$ in Eq. 48 for the correction to the scaling of the entanglement entropy $S_{1}$ in the XX spin chain at zero-magnetic field as function of $X=\ell_{\text {lat }} / L_{\text {lat }}$. Data are reported for two values of $L_{\text {lat }}$ of order of 1000 and 300 which are compatible with the corresponding filling $\nu=1 / 2,1 / 3,1 / 4,1 / 5$. The continuous lines (indistinguishable from data points for not too small $X$ ) are the conjecture in Eq. 49 .

calculations (apart small subleading corrections). As for finite $\alpha$, the smooth result obtained for chains of different length (reported in the left panel) is a consequences of the sampling at integer $\ell_{\text {lat }}$. Using the property of the $\operatorname{Li}_{2}(y)$ function, the envelope in the left panel of Fig. 4 is

$$
F_{\infty}(X)=\frac{(-1)^{\ell_{\text {lat }}}}{4}\left[\operatorname{Li}_{2}\left(-e^{i \pi X}\right)+\operatorname{Li}_{2}\left(-e^{-i \pi X}\right)\right],
$$

that is also shown in both panels, but in the left one is indistinguishable from data points.

3.2.2. The von Neumann entanglement entropy: $\alpha=1$. For $\alpha=1$, the leading correction in Eq. (33) is vanishing. The actual calculation requires a complicated mapping with random matrix theory, but the final result, correct at $O\left(N^{-3}\right)$, can be read from Eq. (34) specialized to $\alpha=1$ :

$d_{1}(N)=-\frac{1}{12 N^{2}}\left(\frac{1}{5}+(\cot \pi \ell / L)^{2}\right)=-\frac{1}{12 N^{2}}\left(\frac{1}{(\sin \pi \ell / L)^{2}}-\frac{4}{5}\right)$.

Fig. 5 (left panel) reports the exact numerical computation for the entanglement entropy for various values of $\ell / N$, showing perfect agreement with the asymptotic formula.

We consider again the XX spin chain for which the corrections to the scaling for the von Neumann entropy have not been considered quantitatively in finite systems. For infinite systems Ref. 21] reports the exact result

$$
d_{1}\left(\ell_{\text {lat }}\right)=-\frac{1}{12 \ell_{\text {lat }}^{2}}\left[\frac{1}{5}+(\cot \pi \nu)^{2}\right] .
$$


It should be pointed out that this term is an "analytical correction" to the scaling, i.e. it is not due to the insertion of an irrelevant operator. For this reason, its finite-size scaling cannot be obtained simply by replacing the distance $\ell_{\text {lat }}$ with the chord length, as done for $d_{\alpha}$ at finite $N$.

For finite systems, we expect the FSS form

$$
d_{1}\left(\ell_{\mathrm{lat}}, L_{\mathrm{lat}}\right)=\frac{1}{\left(\frac{L_{\mathrm{lat}}}{\pi} \sin \left(\pi \ell_{\mathrm{lat}} / L_{\mathrm{lat}}\right)\right)^{2}} F_{1}\left(\ell_{\mathrm{lat}} / L_{\mathrm{lat}}\right),
$$

where $F_{1}(X)$ is an unknown function with $F_{1}(0)$ fixed by Eq. (47). However, by looking at its continuum limit, it is reasonable to propose the FSS ansatz $F_{1}(X)=$ $A+B \sin ^{2} \pi X$. The constant $A$ can be fixed by requiring that $F_{1}(0)$ is given by Eq. 477). The constant $B$ can be fixed with the numerical data (e.g. by the scaling at $X=1 / 2$ ). After a careful analysis, we conjecture the FSS scaling function

$$
F_{1}(X)=-\frac{1}{12}\left(\frac{1}{5}+\cot ^{2} \pi \nu\right)+\frac{1}{18}\left[1+\frac{6}{5} \cot ^{2} \pi \nu\right] \sin ^{2}(\pi X) .
$$

By construction, this form reproduces Eq. 477) for $X=0$. In the continuum limit, i.e. $\nu \rightarrow 0$, it reproduces Eq. 46), as an highly non-trivial check. A stringent test of its correctness is provided by numerical data. These are reported for few different values of $\nu$ and for large values of $L_{\text {lat }}$ in Fig. 5 right panel, showing a perfect agreement.

\section{Excited states in periodic chains}

We now turn our attention to excited states that can be easily treated in the formalism we introduced. Indeed, the only change compared to the ground state is in Eq. (16) where we have to sum over the occupied one-particle levels. It is convenient to have a simple graphical representation of the many-body states. This can be easily done by representing each single particle state with a circle and filling in black the occupied ones and leaving empty the others. For example, the ground state is

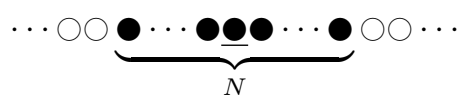

where the underlined circle represents the zero momentum mode. When working at fixed number of particles $N$, excited states are obtained from the ground states just by moving black circles to empty white ones.

The entanglement of excited states has been already considered few times in the literature, but only in the context of discrete lattice models. In [4] it was shown that the negativity (which is a different measure of entanglement, related to some Rényi entropies [1]) shows a universal scaling in critical spin systems. In Ref. [45, on the bases of Toeplitz matrix arguments for the XX spin chain and by exact calculations for the anisotropic Heisenberg one, it has been shown that only a small subclass of excited states can exhibit a universal logarithmic divergence with the subsystem size $\ell$, while most of the states strongly violates the area law and their entanglement entropies increase linearly with $\ell$, with non-universal prefactor. The states providing universal scaling are those where there is a finite (and possibly small) number of sets of one-particle states occupied sequentially, as e.g.

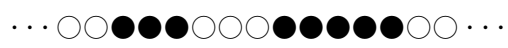


and the locations of these blocks of states is not essential. This set of states includes all low-lying excited states. In Ref. [46] it has been shown that the entanglement entropies of low-lying excited states display a universal finite size scaling that is different from the one in the ground state of Eq. (3). These can be calculated by means of CFT, because low-lying states in CFT language are described by the action of a scaling operator on the ground state. The states that are obtained by applying a primary operator to the ground states are of particular importance. In this case, the Rényi entropies for integer $\alpha$ have been related to the correlation functions of these operators in a $\alpha$-sheeted Riemann surface [46]. We remand the interested reader to the original reference [46] and we limit to quote the main result

$\operatorname{Tr} \rho_{A}^{\alpha}=\left[\operatorname{Tr} \rho_{A}^{\alpha}\right]_{(G S)} F_{\Upsilon}^{(\alpha)}(\ell / L)=c_{\alpha}\left(\frac{L}{\pi} \sin (\pi \ell / L)\right)^{c / 6(\alpha-1 / \alpha)} F_{\Upsilon}^{(\alpha)}(\ell / L)$.

where $\left[\operatorname{Tr} \rho_{A}^{\alpha}\right]_{(G S)}$ is the ground-state value. $F_{\Upsilon}^{(\alpha)}(X)$ is the universal scaling function depending on the operator $\Upsilon$ whose action on the ground state gives the desired excited state. In particular $F_{\Upsilon}^{(\alpha)}(0)=1$, i.e., in the thermodynamic limit, all these low-lying states have entropies degenerate with the ground-state, in agreement with Ref. [45.

Two sets of primary operators can be easily treated for a free boson compactified on a circle, which describes the thermodynamic limit of the free-fermion gas we are considering. First, the vertex operators $V(x)$ for which Ref. [46] reports $F_{V}^{(\alpha)}(X)=1$ (i.e., the entanglement entropies are the same as in the ground-state). In the freefermion gas, this corresponds to the excited states obtained by shifting the groundstate (50) in momentum space, i.e. replacing all $k_{i}$ with $k_{i}+M$ with $M$ arbitrary integer number. The matrix $\mathbb{A}$ is always given by Eq. (28), that depends only of the differences between the various momenta, and so it is exactly equal to the ground-state one, confirming the prediction $F_{V}^{(\alpha)}(X)=1$.

The other operator considered in Ref. [46] is $\Upsilon=i \partial \phi$, which has been found to have a non-trivial scaling function given by

$$
F_{\Upsilon}^{(\alpha)}(X)=(-1)^{\alpha}\left(\frac{2}{\alpha} \sin (\pi X)\right)^{2 \alpha} \operatorname{det} \mathbb{H},
$$

for integer $\alpha$. Here $\mathbb{H}$ is a $2 \alpha \times 2 \alpha$ matrix with elements

$$
\mathbb{H}_{j k}=\left\{\begin{array}{ll}
\frac{1}{e^{i z_{j}}-e^{i z_{k}}} & \text { if } j \neq k \\
0 & \text { if } j=k
\end{array} \text { and } \quad z_{j}=\left\{\begin{array}{ll}
\pi(2 j-2+x) / \alpha & \text { if } j \leq \alpha \\
\pi(2 j-2-x) / \alpha & \text { if } j>\alpha
\end{array}\right. \text {. }\right.
$$

For $\alpha=2$, this reduces to the simple expression

$$
F^{(2)}(X)=\frac{(7+\cos (2 \pi X))^{2}}{64},
$$

but for any other $\alpha>2$ the explicit formulas are too cumbersome to be reported in their full glory. It must be mentioned that the analytic continuation of $F_{\Upsilon}^{(\alpha)}(X)$ is not yet known, and so also the von Neumann entanglement entropy of this excited state is still unknown. However for small $X$ it has been found

$$
F_{\Upsilon}^{(\alpha)}(X)=1-\frac{(\pi X)^{2}}{3}\left(\alpha-\alpha^{-1}\right)+O\left(X^{3}\right),
$$

whose analytic continuation is obvious. 


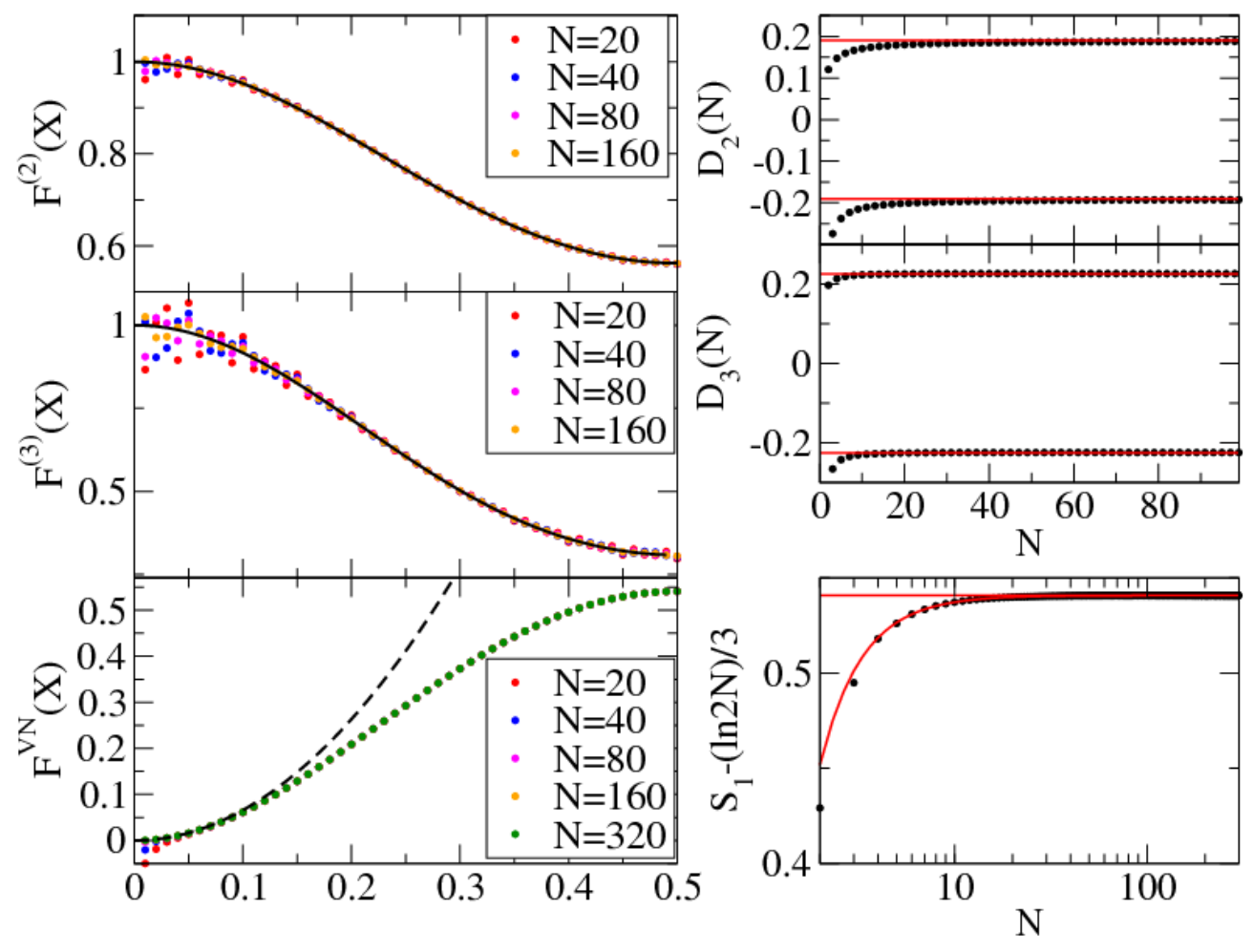

Figure 6. Scaling functions for the entanglement Rényi entropies in the lowest energy particle-hole excited state of a periodic system in Eq. (57). Left top panels report the scaling function $F^{(\alpha)}(X)$ for $\alpha=2,3$ as function of $X$, showing good agreement with the CFT prediction (53) shown as continuous lines. The lower left panel reports the scaling function for the von Neumann entropy that for small $X$ agrees with the general expansion (56), reported as a dashed line. On the right the universal corrections to the scaling are reported for the half-system entanglement showing the behavior $N^{-2 / \alpha}$ for the leading corrections. For $\alpha=1$, the corrections are monotonic (last panel) and effectively described by Eq. 62.

The excited state generated by the action of $i \partial \phi$ on the ground state is the particle-hole excitation obtained by moving one particle from the highest occupied level to the first available one, i.e., graphically

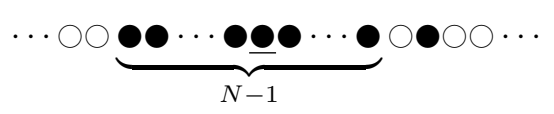

The corresponding $N \times N$ matrix $\mathbb{A}$ has then the first $n-1$ rows and columns identical to the ground state Eq. 29 , but the last different, given by

$$
\mathbb{A}_{N m}=\left\{\begin{array}{ll}
\frac{\sin \pi(N+1-m) \ell / L}{\pi(N+1-m)}, & m=1, \ldots, N-1 \\
\ell / L & m=N
\end{array},\right.
$$

and $\mathbb{A}_{m N}=\mathbb{A}_{N m}$. Despite only one row and one column differ from the ground-state, the matrix $\mathbb{A}$ ceases to be a Toeplitz matrix and (to the best of our knowledge) no analytic treatment is possible anymore. 
We check the prediction in Eq. 53 numerically. In Fig. 6, we report the numerical calculated scaling function

$F^{(\alpha)}(X) \equiv \exp \left[(1-\alpha)\left(S_{\alpha}(N)-(1+1 / \alpha) / 6 \ln (2 N \sin \pi X)-E_{\alpha}\right)\right]$,

for several values of $N$ as a function of $X$ for $\alpha=2,3$. It is evident that in the large $N$ limit the CFT prediction (53) is approached with small oscillating corrections to the scaling which are more pronounced for small $X$. In order to shed some light on the analytic continuation at $\alpha \rightarrow 1$, we also report (always in Fig. 6) the scaling function for the von Neumann entropy

$$
F^{V N}(X) \equiv S_{1}(N)-1 / 3 \ln (2 N \sin \pi X)-E_{1} .
$$

As a difference with $F^{(\alpha)}$ with $\alpha \geq 2$, the corrections to the scaling are much smaller, as for the entanglement in the ground-state. Unfortunately, as already stated, the analytic continuation to $\alpha \rightarrow 1$ of Eq. (53) for arbitrary $X$ is not yet known and so the data in the figure cannot be contrasted to an exact prediction. However, such an analytic continuation is known for small $X$ : from Eq. (56) we have $F^{V N}(X)=2(\pi X)^{2} / 3+O\left(X^{3}\right)$. This prediction is reported on top of the numerical data and they agree perfectly up to $X \sim 0.1$.

Having established the leading asymptotic behavior, we move our interest to the leading corrections to the scaling. We find numerically that the corrections have the same exponents as in the ground-state, i.e. they decay with $\alpha$ dependent power-law $N^{-2 / \alpha}$. In order to show this, we report in Fig. 6] (left panels) the quantity

$$
D_{\alpha}(N)=\left[S_{\alpha}(N)-S_{\alpha}^{\text {asy }}(N)\right] N^{2 / \alpha},
$$

for the half-system entanglement. The data clearly show the behavior for the corrections of the form $(-1)^{N} N^{-2 / \alpha}$ for $\alpha>1$. We found numerically that $D_{2} \sim(-1)^{N} 0.19039 \ldots$ and $D_{3} \sim(-1)^{N} 0.225 \ldots$ These non-universal amplitude are different from the ones found for the ground state and we have been not able neither to calculate nor to guess their $\alpha$ dependence. We checked that for general $\ell / L$, the corrections are of the form $\cos (2 \pi N \ell / L) N^{-2 / \alpha}$ as for the ground state. Furthermore subleading corrections seem to have the same power structure as in the ground-state (cf. Eq. (34)).

The von Neumann entropy at $\alpha=1$ requires a separate analysis. Indeed, as the last panel of Fig. 66 shows, the correction to the scaling are monotonic. However in this case we do not know exactly the constant term in the leading behavior. An accurate numerical analysis for the half-system entanglement is consistent with the behavior

$$
S_{1}(N)=\frac{1}{3} \log 2 N+y_{1}+\frac{y_{2}}{N^{2}},
$$

with $y_{1}=0.540726 \ldots$ and $y_{2}=0.35 \ldots$ These numerical data have been obtained by fitting data for $N>100,150,200$ and keeping under control the stability of the fit. Although these fitting parameters have been extracted from asymptotic large $N$, Fig. 6 shows that the fit describes very accurately the data down to $N \sim 3$.

\section{Systems with boundaries}

\subsection{Hard-wall boundaries}

We now consider a gas of spinless fermions confined in the interval $[0, L]$ by a hardwall potential, i.e. the gas density vanishes outside the interval $x \notin[0, L]$ and the 

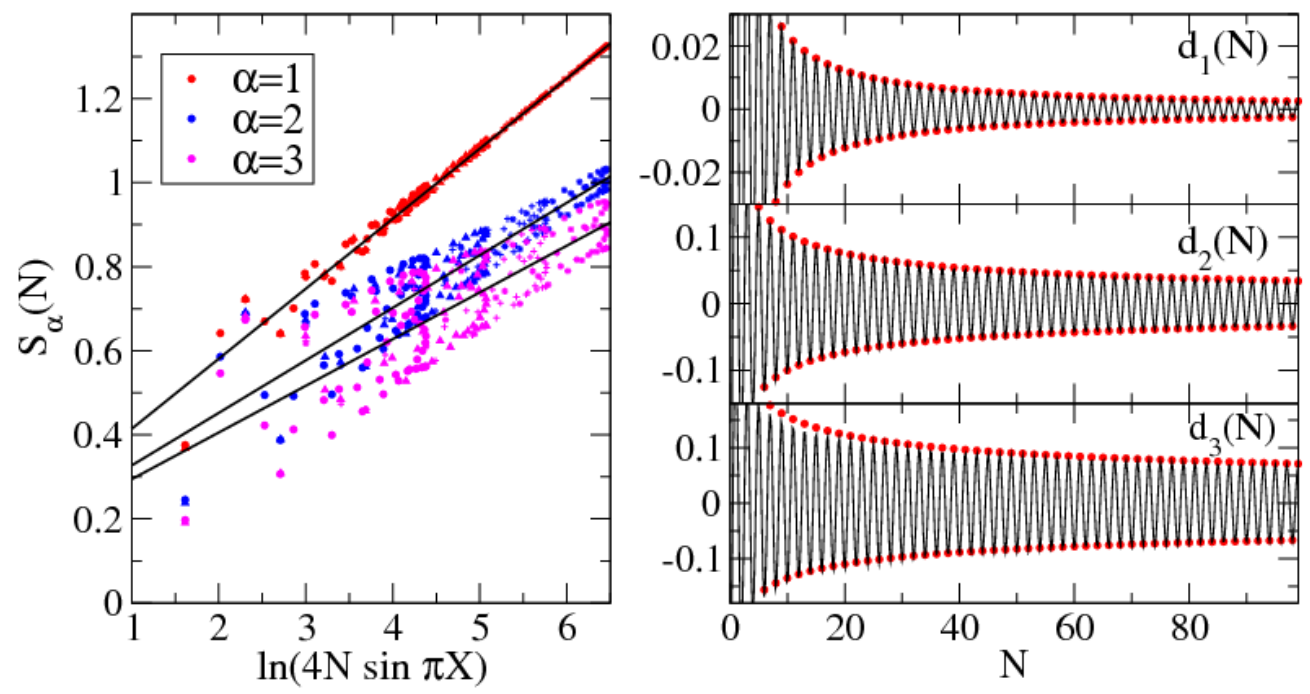

Figure 7. On the left we report the Rényi entanglement entropies $S_{\alpha}$ for the free-fermion gas confined by hard wall potential for $\alpha=1,2,3$ (from top to bottom) as function of $\ln (4 N \sin \pi X)$ with $X=\ell / L$. For the larger values of $\alpha$, oscillating corrections to the scaling obscure the leading asymptotic behavior Eq. 65 represented as straight lines. These oscillating corrections are quantitatively described by Eq. 67 that is checked in the right panel for the same values of $\alpha$ for the half-system entanglement $(\ell=L / 2)$.

boundary condition is that the wave-function vanishes at the boundaries (Dirichlet $\mathrm{BC})$. The one-particle wave functions are

$$
\phi_{k}(x)=\sqrt{\frac{2}{L}} \sin \left[\pi k \frac{x}{L}\right], \quad k=1,2, \ldots,
$$

with energies $E_{k}=\pi^{2} k^{2} / 2 L^{2}$.

5.1.1. An interval starting from the boundary. The elements of the overlap matrix (cf. Eq. (23p) between two one-particle eigenstates $n$ and $m$ have a particularly simple form for an interval starting from the boundaries, i.e. $A=[0, \ell]$. In fact we have

$\mathbb{A}_{n m}=\int_{0}^{\ell} d z \phi_{n}^{*}(z) \phi_{m}(z)=\mathbb{B}_{n m}(\ell) \equiv \frac{\sin [\pi(n-m) \ell / L]}{\pi(n-m)}-\frac{\sin [\pi(n+m) \ell / L]}{\pi(n+m)}$.

with $n, m=1, \ldots, N$.

As for the periodic case, the matrix $\mathbb{A}$ above is exactly the same as the correlation matrix $\mathbb{C}^{\text {lat }}$ of an infinite lattice with $\nu$ replaced by $\ell / N$. This has been considered in Ref. 43 where, using a recent generalization of the Fisher-Hartwig conjecture to Toeplitz+Henkel matrices [4], the asymptotic behavior of the entanglement entropies for the lattice model have been calculated exactly. Exploiting the equivalence between the two problems (i.e. replacing $\nu$ with $\ell / N$ in Ref. [43]) we easily obtain for the asymptotic behavior of the entanglement entropies

$$
S_{\alpha}=\frac{1}{12}\left(1+\frac{1}{\alpha}\right) \ln [2(2 N+1) \sin \pi X]+\frac{E_{\alpha}}{2}+\cdots,
$$


where $E_{\alpha}$ is defined in Eq. (11). Notice that this result agrees with the general CFT prediction in Eq. (4) with $\ln g=0$ that is a well-known result for open boundary conditions [10.

A comparison of the finite- $N$ results with Eq. (65) is shown in Fig. 7. It is evident that for any $\alpha$ there are corrections to the scaling oscillating with $N$. These are of the order $O\left(N^{-1 / \alpha}\right)$ and can be deduced exactly from the analogy with the lattice model solved in Ref. [43. Defining

$$
d_{\alpha}(N)=S_{\alpha}(N)-S_{\alpha}^{\text {asy }}(N),
$$

we have from Ref. [43] and replacing $\nu$ with $\ell / L$

$d_{\alpha}(N)=\frac{2 \sin [\pi(2 N+1) \ell / L]}{1-\alpha}[2(2 N+1) \sin \pi \ell / L]^{-1 / \alpha} \frac{\Gamma\left(\frac{1}{2}+\frac{1}{2 \alpha}\right)}{\Gamma\left(\frac{1}{2}-\frac{1}{2 \alpha}\right)}$.

Fig. 7 (right panel) show these corrections for half-system entanglement entropy for $\alpha=1,2,3$. Further corrections of the form $N^{-p / \alpha}$ with $p$ integer can be straightforwardly deduced from the analysis in Ref. [43. We mention that, as for the periodic case, these leading $N^{-1 / \alpha}$ corrections correspond to the ones of the form $O\left(\ell^{-1 / \alpha}\right)$ found within CFT [37, 43].

5.1.2. Generic interval. In the case of a subsystem consisting of generic interval $A=\left[x_{1}, x_{2}\right]$, the entanglement entropies require a different analysis. The general formula for the matrix $\mathbb{A}$ is slightly more complicated:

$$
\mathbb{A}_{n m}=\int_{x_{1}}^{x_{2}} d z \phi_{n}^{*}(z) \phi_{m}(z)=\mathbb{B}_{n m}\left(x_{2}\right)-\mathbb{B}_{n m}\left(x_{1}\right),
$$

where the matrix $\mathbb{B}$ is defined in Eq. (64). The entanglement entropies of the interval $\left[x_{1}, x_{2}\right]$ can be computed by inserting its eigenvalues in Eq. (26). This allows us to easily compute $S_{\alpha}(N)$ up to large values of $N$, and compare its behavior with the asymptotic CFT prediction

$S_{\alpha}(N)=\frac{1}{6}\left(1+\frac{1}{\alpha}\right)\left\{\ln 4 N+E_{\alpha}+\frac{1}{2} \ln \left[\frac{\sin ^{2}\left[\pi\left(y_{2}-y_{1}\right) / 2\right] \sin \left(\pi y_{1}\right) \sin \left(\pi y_{2}\right)}{\sin ^{2}\left[\pi\left(y_{2}+y_{1}\right) / 2\right]}\right]\right\}$,

where $y_{i}=x_{i} / L$. The proof of this equation is a straightforward CFT exercise that we report in Appendix A

In particular, we considered a block of size $L y$ centered at the middle of the system, i.e., $y_{2}=1 / 2+y / 2$ and $y_{1}=1 / 2-y / 2$. The data of the entanglement entropy approach the asymptotic behavior predicted by Eq. 69, , i.e.,

$$
S_{\alpha}(N)=\frac{1}{6}\left(1+\frac{1}{\alpha}\right)\left[\ln 2 N+E_{\alpha}+\ln \sin (\pi y)\right],
$$

as shown for $\alpha=1$ in Figs. 8 (left panel). It can be seen numerically that the corrections to the scaling are of the order $O\left(N^{-1 / \alpha}\right)$. It must be noted however that the $O\left(N^{-1 / \alpha}\right)$ convergence is nonuniform approaching the limits $x \rightarrow 0$ and $x \rightarrow 1$, where the entanglement entropy trivially vanishes for any $N$. Simpler results are obtained also considering an interval starting from the center, i.e. taking $x_{2}=1 / 2 \pm y$ and $x_{1}=1 / 2$, for which

$$
S_{\alpha}(N)=\frac{1}{6}\left(1+\frac{1}{\alpha}\right)\left\{\ln 4 N+E_{\alpha}+\frac{1}{2} \ln \left[\cos (\pi y) \tan (\pi y / 2)^{2}\right]\right\},
$$

see Fig. 8 (right panel) for $\alpha=1$. We also checked the correctness of Eq. (69) for other values of $\alpha$ and for different choices of $x_{1}$ and $x_{2}$, but the resulting figures are not very illuminating and we do not report them. 

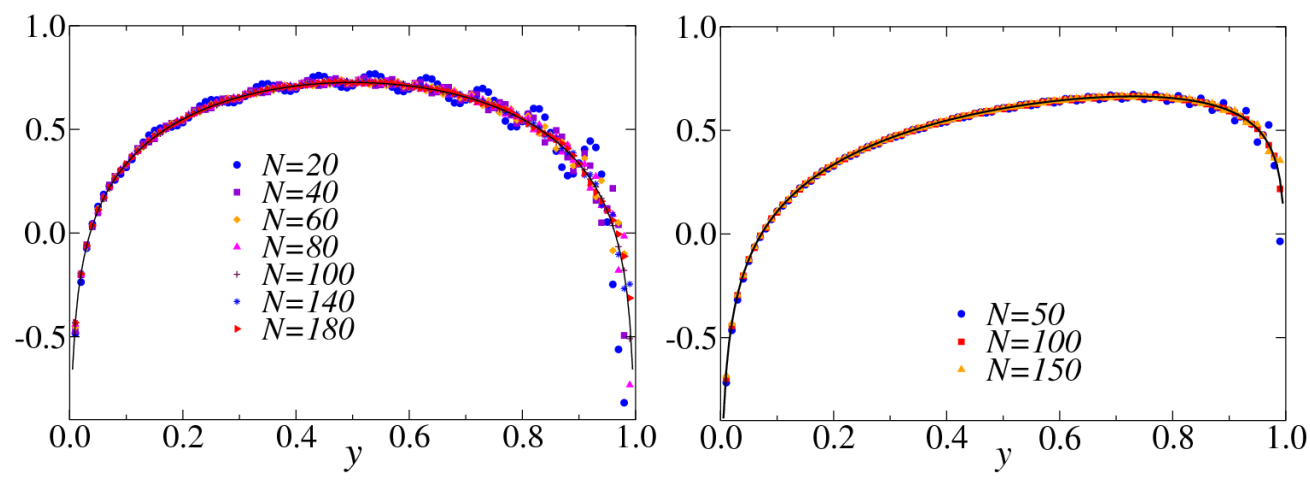

Figure 8. (Color online) The von Neumann entanglement entropies $S_{1}$ of intervals $\left[x_{1}, x_{2}\right]$. In particular we consider the cases $x_{1}=L(1 / 2-y / 2)$, $x_{2}=L(1 / 2+y / 2)$ (left) and $x_{1}=L / 2, x_{2}=L(1 / 2+y / 2)$ (right). We plot $S_{1}(N)-(\ln N) / 6$ against $y$. In both figures the black full line shows the asymptotic behavior given by Eq. 69p.

\subsection{Neumann boundary conditions}

Another interesting situation arise when imposing Neumann boundary conditions on the fermionic wave-function, i.e. imposing that the derivative of the wave function vanishes at the two boundaries at 0 and $L$. In this case, the normalized one-particle wave functions are

$$
\phi_{k}(x)=\sqrt{\frac{2-\delta_{k, 0}}{L}} \cos \left[\frac{\pi k x}{L}\right], \quad k=0,1, \ldots,
$$

with the same energy as for Dirichlet BC. As an important difference compared to Dirichlet $\mathrm{BC}$, also the zero-mode with $k=0$ does contribute.

The $\mathbb{A}$ matrix is readily calculated. It is an $N$-by- $N$ matrix with entries that are more easily written if we count rows and columns with $n, m$ starting from 0 and up to $N-1$ as for the modes above. For an interval of length $\ell$ starting from the boundary, straightforward calculations lead to

$\mathbb{A}_{n m}= \begin{cases}\frac{\sin [\pi(n-m) z]}{\pi(n-m)}+\frac{\sin [\pi(n+m) z]}{\pi(n+m)} & \text { if } n, m=1, \ldots, N-1, \\ \sqrt{2} \frac{\sin [\pi m z]}{\pi m} & \text { if } n=0 \text { and } m \neq 0, \\ z & \text { if } m, n=0,\end{cases}$

and $\mathbb{A}_{0 m}=\mathbb{A}_{m 0}$. Note the plus sign between the two terms for $n, m \neq 0$ and the zero-mode contribution, as a difference compared to Dirichlet BC.

Because of the presence of the zero row and column, $\mathbb{A}$ is not of the form Toeplitz+Hankel as it is for Dirichlet BC. Thus the recent generalizations of FisherHartwig conjecture in Ref. [47] cannot be used. We then determine numerically the matrix $\mathbb{A}$ for various $N$ and, through Eq. $(26)$, we compute the Rényi entanglement entropy shown in Fig. 9 (left panel). The analysis of their large- $N$ behavior gives

$$
S_{\alpha}(N)=\frac{1}{12}\left(1+\frac{1}{\alpha}\right) \ln [2(2 N-1) \sin (\pi \ell / L)]+\frac{E_{\alpha}}{2}+\cdots,
$$

shown as continuous lines in the figure. This form is consistent with the general CFT expectation in Eq. (4) with $g=1$. In order to avoid confusion with CFT literature, 

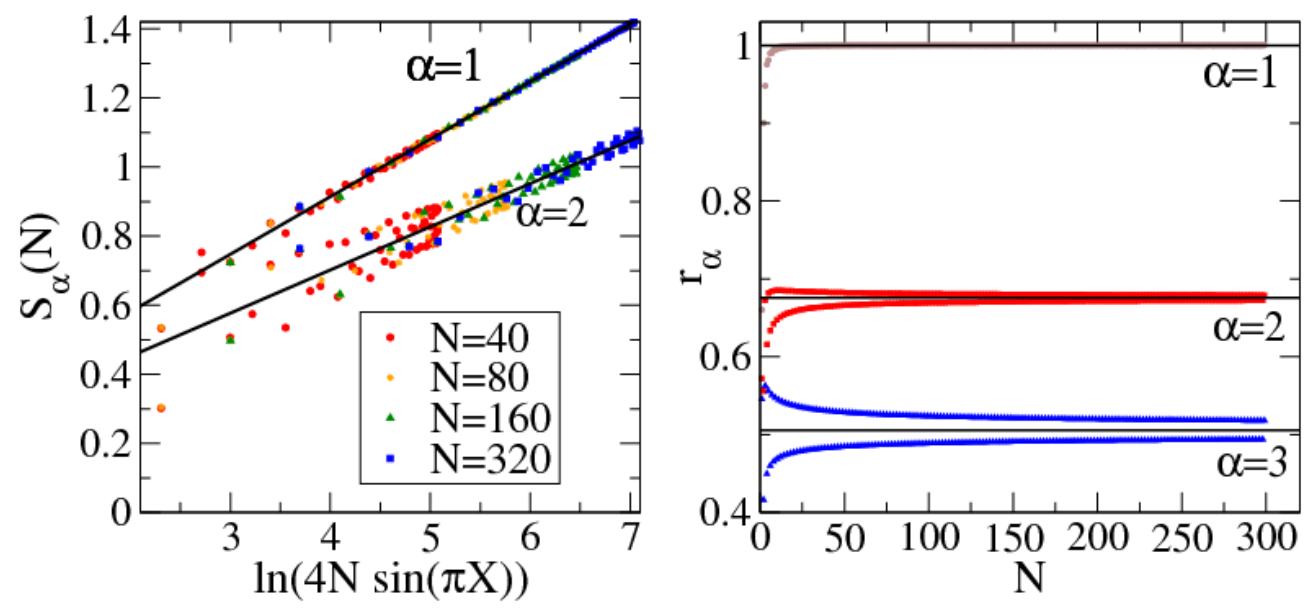

Figure 9. On the left we report the Rényi entanglement entropies $S_{\alpha}$ for the free-fermion gas with Neumann BC for $\alpha=1,2$ (from top to bottom) as function of $\ln (4 N \sin \pi X)$ with $X=\ell / L$. Oscillating corrections to the scaling are evident on top of the leading asymptotic behavior Eq. 74 represented as straight lines. These oscillating corrections are quantitatively described by Eq. 75 that is checked in the right panel, where we report the quantity $r_{\alpha}$ in Eq. 76 for $\alpha=1,2,3$ against the theoretical prediction from Eq. 75 for the half-system entanglement $(\ell=L / 2)$.

we stress that, in this paper, we are considering Neumann and Dirichlet BC on the fermion degrees of freedom. These do not correspond to Dirichlet and Neumann BC on the bosonic field obtained from the bosonization of the fermionic theory that instead are well known to have different $g$ function (see e.g. 48]). It is known that they both correspond to Neumann conditions of the bosonized field and so it should be not a surprise that the asymptotic behavior up to $O\left(N^{0}\right)$ is the same as Eq. 65). Notice that we have included a $O(1 / N)$ term in the leading behavior of the logarithm (i.e. the -1 in $(2 N-1))$ that has the effect to cancel the leading non-oscillating correction to the scaling. This was present also for Dirichlet BC, but it has opposite sign. While before this was motivated by the mapping to the lattice model (cf. Ref. [4]), here we introduced it on a phenomenological basis in order to describe the data (see below) and we do not have any mathematical explanation for it.

We now consider the corrections in $N$ to the leading behavior, that are again consistent with the general scaling from CFT $O\left(N^{-1 / \alpha}\right)$. On the basis of the numerics, we guess exactly the first correction to the scaling, and we can write the entanglement entropies as

$$
\begin{aligned}
S_{\alpha}(N)= & \frac{1}{12}\left(1+\frac{1}{\alpha}\right) \ln [2(2 N-1) \sin (\pi \ell / L)]+\frac{E_{\alpha}}{2}+ \\
& +\frac{2 \sin [\pi(2 N-1) \ell / L]}{1-\alpha}[2(2 N-1) \sin \pi \ell / L]^{-1 / \alpha} \frac{\Gamma\left(\frac{1}{2}+\frac{1}{2 \alpha}\right)}{\Gamma\left(\frac{1}{2}-\frac{1}{2 \alpha}\right)} .
\end{aligned}
$$

In Fig. 9 (right panel) we show the evidences for this scaling for the half-system entanglement $(\ell=L / 2)$. We report the quantity

$$
r_{\alpha} \equiv \frac{\left|S_{\alpha}(N)-\frac{1}{6} \ln (2(2 N-1))-E_{\alpha} / 2\right|}{[2(2 N-1)]^{-1 / \alpha}},
$$


that for $\alpha=1,2,3$ approaches for large $N$ the value predicted from the ansatz 75 $r_{\alpha}=\frac{2}{1-\alpha} \frac{\Gamma\left(\frac{1}{2}+\frac{1}{2 \alpha}\right)}{\Gamma\left(\frac{1}{2}-\frac{1}{2 \alpha}\right)}$. For $\alpha=1$ the leading corrections are of order $1 / N$. The figure shows that the absolute value for even and odd $N$ coincide (but they have opposite signs). This confirms that choosing to parametrize the leading term with $(2 N-1)$ in Eq. 74 cancels the $1 / N$ non-oscillating corrections completely. Some of the factors $(2 N-1)$ in Eq. 75 are subleading and are not explicitly tested by the numerical data presented. They have been introduced from the analogy with Dirichlet BC results. Finally, we stress that we do not have any mathematical basis to justify Eq. (75): while its general structure can be inferred by CFT [37] because fermionic Neuman BC are in the same universality class as Dirichlet BC, the non-universal amplitude of the correction has been guessed and conjectured by exploiting the analogy with Dirichlet $\mathrm{BC}$ and tested agains numerical data.

We also considered numerically other situations, such as other bipartitions of the systems. However, none of these results present particularly relevant or unexpected features to be mentioned here.

\section{Conclusions}

In this manuscript we report the details about the computation of the entanglement entropies of continuous systems (gases) which have been anticipated in the short communication [19]. The most important ingredient to write down the entanglement entropies in terms of finite determinants is the use of the reduced overlap matrix in Eq. 23. The calculation of the entanglement entropies is then mapped to the solution of an eigenvalue problem of an $N \times N$ matrix, with $N$ being the number of particles of the gas.

For the ground state of a periodic system we obtain the leading behavior in the form

$$
S_{\alpha}=\frac{1}{6}\left(1+\frac{1}{\alpha}\right) \ln \left(2 N \sin \pi \frac{\ell}{L}\right)+E_{\alpha}+o\left(N^{0}\right),
$$

while for a gas with Dirichlet or Neumann boundary conditions we find

$$
S_{\alpha}=\frac{1}{12}\left(1+\frac{1}{\alpha}\right) \ln \left(4 N \sin \pi \frac{\ell}{L}\right)+\frac{E_{\alpha}}{2}+o\left(N^{0}\right),
$$

both in agreement with CFT and scaling expectations, but they have been found here from first principles. We also derive the corresponding leading behavior for some classes of excited states.

Furthermore, adapting to the problem at hand the results in Refs. [21, 27], we calculate also subleading corrections. The universality of these formulas allowed us to infer the finite-size scaling forms for spin chains which are reported in Eq. (40) for $1<\alpha<\infty$, in Eq. 42 for $\alpha=\infty$, and in Eq. 49 for $\alpha=1$. The determination of these exact formulas were left as open problems from previous investigations.

Some other applications (such as to systems with defects, star graphs, and to gases confined by an external potential both in and out of equilibrium) of this novel method have been already shortly presented in Ref. [19], but they will be detailed elsewhere. Other generalizations, which we are currently investigating, concern the calculation of the entanglement for quadratic Hamiltonian which do not conserve the fermion number (such as the continuum limit of the XY model), free gases in higher dimensions and different geometries. Finally, some non equilibrium situations such as 
local quantum quenches (e.g., instantaneously turning on/off of a defect) can also be tackled within this framework. The asymptotic CFT results in several circumstances are known [51, but analytic calculations for specific models are still missing. They may provide important insights in view of the recent proposals of using the full counting statistics after a quench as an experimental probe and a measure of entanglement [52].

\section{Acknowledgments}

We thank Maurizio Fagotti and Fabian Essler for helpful discussions. We thank German Sierra and Miguel Ibanez Berganza for correspondence about Ref. [46].

\section{Appendix A. The CFT entanglement entropy of an arbitrary interval in a finite system with Dirichlet boundary conditions}

We provide here the CFT proof of Eq. (69). In CFT the moments of the reduced density matrix can be written as correlation function of particular twist fields that transform as primary operators under a conformal transformation 6, 7, 49. In particular, for the case of a finite interval between $x_{1}$ and $x_{2}$ in a boundary theory, we have (for integer $n$ )

$$
\operatorname{Tr} \rho_{A}^{n}=\left\langle\Phi_{n}\left(x_{1}\right) \Phi_{-n}\left(x_{2}\right)\right\rangle,
$$

where $\Phi_{n}$ and $\Phi_{-n}$ transform as primary fields with dimensions $x_{n}=(c / 24)(n-1 / n)$.

This two-point function in the finite strip with $\operatorname{Re}(w) \in[0, L]$ can be obtained from its conformal mapping to the upper half plane (UHP) with $\operatorname{Im}(z)>0$. The mapping and its inverse are

$$
i w=\frac{L}{\pi} \ln z, \quad z=e^{i \pi w / L} .
$$

Using the property that the twist fields for free fermions with open boundary conditions behaves like primary operators in a free bosonic theory [43, the two-point function in the UHP can be read from Ref. 50]

$$
\left\langle\Phi_{n}\left(z_{1}\right) \Phi_{-n}\left(z_{2}\right)\right\rangle_{\mathrm{UHP}}=c_{n}\left(\frac{z_{1 \overline{2}} z_{2 \overline{1}}}{z_{12} z_{\overline{1} \overline{2}} z_{1 \overline{1}} z_{2 \overline{2}}}\right)^{2 x_{n}}
$$

with $z_{i j}=\left|z_{i}-z_{j}\right|$ and $z_{\bar{k}}=\overline{z_{k}}$ and $c_{n}$ an undetermined constant (we set the UV cut-off $a$ to 1). We stress that in general (i.e. for a theory that is not free bosonic) a universal function of the harmonic ratio build with the four points $z_{i}$ and $\bar{z}_{i}$ multliplies the above formula 31,32 . Using then the conformal mapping $(\mathrm{A} .22$, we have

$$
\begin{aligned}
& z_{12} z_{\overline{1} \overline{2}}=\left(e^{i \pi w_{1} / L}-e^{i \pi w_{2} / L}\right)\left(e^{-i \pi w_{1} / L}-e^{-i \pi w_{2} / L}\right)=4 \sin ^{2} \frac{\pi\left(w_{1}-w_{2}\right)}{2 L}, \\
& \left|z_{1 \overline{1}}\right|=2 \sin \frac{\pi w_{1}}{L}, \quad\left|z_{2 \overline{2}}\right|=2 \sin \frac{\pi w_{2}}{L}, \\
& z_{1 \overline{2}} z_{\overline{1} 2}=\left(e^{i \pi w_{1} / L}-e^{-i \pi w_{2} / L}\right)\left(e^{-i \pi w_{1} / L}-e^{i \pi w_{2} / L}\right)=4 \sin ^{2} \frac{\pi\left(w_{1}+w_{2}\right)}{2 L} .
\end{aligned}
$$

Thus

$$
\begin{aligned}
\left\langle\Phi_{n}(r, \tau) \Phi_{-n}(0, \tau)\right\rangle_{\text {strip }} & =\left|w^{\prime}\left(z_{1}\right)\right|^{-x_{n}}\left|w^{\prime}\left(z_{2}\right)\right|^{-x_{n}}\left\langle\Phi_{n}\left(z_{1}(w)\right) \Phi_{-n}\left(z_{2}(w)\right)\right\rangle_{\mathrm{UHP}}= \\
& =c_{n}\left(\frac{\pi^{2}}{4 L^{2}} \frac{\sin ^{2} \frac{\pi\left(w_{1}+w_{2}\right)}{2 L}}{\sin ^{2} \frac{\pi\left(w_{1}-w_{2}\right)}{2 L} \sin \frac{\pi w_{1}}{L} \sin \frac{\pi w_{2}}{L}}\right)^{2 x_{n}} .
\end{aligned}
$$


Using Eqs. A.1 and (11) performing the analytic continuation from $n$ to $\alpha$, we have

$S_{\alpha}=\frac{c}{6}\left(1+\frac{1}{\alpha}\right)\left[\ln \frac{2 L}{\pi}+\frac{1}{2} \ln \frac{\sin ^{2} \frac{\pi\left(w_{1}-w_{2}\right)}{2 L} \sin \frac{\pi w_{1}}{L} \sin \frac{\pi w_{2}}{L}}{\sin ^{2} \frac{\pi\left(w_{1}+w_{2}\right)}{2 L}}\right]+E_{\alpha}$,

where the constant $E_{\alpha}$ is given by Eq. 111), according to the result for the single interval in a periodic systems. Assuming now the scaling hypothesis when working with finite number of particles, Eq. (69) follows simply by replacing $L / \pi$ by $2 N$ using the argument in Section 1.1 .

\section{References}

[1] L. Amico, R. Fazio, A. Osterloh, and V. Vedral, Entanglement in many-body systems, Rev. Mod. Phys. 80, 517 (2008); J. Eisert, M. Cramer, and M. B. Plenio, Area laws for the entanglement entropy - a review, Rev. Mod. Phys. 82, 277 (2010). Entanglement entropy in extended systems, P. Calabrese, J. Cardy, and B. Doyon Eds, J. Phys. A 42, 500301 (2009).

[2] J. Eisert and M. Cramer, Single-copy entanglement in critical spin chains, Phys. Rev. A 72, 42112 (2005); I. Peschel and J. Zhao, On single-copy entanglement, J. Stat. Mech. P11002 (2005); R. Orus, J.I. Latorre, J. Eisert, and M. Cramer, Half the entanglement in critical systems is distillable from a single specimen, Phys. Rev. A 73, 060303 (2006).

[3] J. Cardy, The ubiquitous 'c': from the Stefan-Boltzmann law to quantum information, J. Stat. Mech. (2010) P10004.

[4] C. Holzhey, F. Larsen, and F. Wilczek, Geometric and renormalized entropy in conformal fieldtheory, Nucl. Phys. B 424, 443 (1994).

[5] G. Vidal, J. I. Latorre, E. Rico, and A. Kitaev, Entanglement in quantum critical phenomena, Phys. Rev. Lett. 90, 227902 (2003); J. I. Latorre, E. Rico, and G. Vidal, Ground state entanglement in quantum spin chains, Quant. Inf. Comp. 4, 048 (2004).

[6] P. Calabrese and J. Cardy, Entanglement entropy and quantum field theory, J. Stat. Mech. P06002 (2004).

[7] P. Calabrese and J. Cardy, Entanglement entropy and conformal field theory, J. Phys. A 42, 504005 (2009).

[8] H-Q Zhou, T Barthel, J O Fjaerestad, and U Schollwoeck, Entanglement and boundary critical phenomena, Phys. Rev. A 74, 050305 (2006).

[9] N Laflorencie, E S Sorensen, M-S Chang, and I Affleck, Boundary effects in the critical scaling of entanglement entropy in 1D systems, Phys. Rev. Lett. 96, 100603 (2006).

[10] I Affleck and A W W Ludwig, Universal non-integer ground-state degeneracy in critical quantum systems, Phys. Rev. Lett. 67, 161 (1991).

[11] P Calabrese and A Lefevre, Entanglement spectrum in one-dimensional systems, Phys. Rev. A 78, 032329 (2008).

[12] L Tagliacozzo, T R. de Oliveira, S Iblisdir, and J I Latorre, Scaling of entanglement support for Matrix Product States, Phys. Rev. B 78, 024410 (2008); F Pollmann, S Mukerjee, A M Turner, and J E Moore, Theory of finite-entanglement scaling at one-dimensional quantum critical points, Phys. Rev. Lett. 102, 255701 (2009).

[13] J.I. Cirac and F. Verstraete, Renormalization and tensor product states in spin chains and lattices, J. Phys. A 42, 504004 (2009); U. Schollwoeck, The density-matrix renormalization group in the age of matrix product states Ann. Phys. 326, 96 (2011).

[14] F. Verstraete and J.I. Cirac, Continuous Matrix Product States for Quantum Fields, Phys. Rev. Lett. 104, 190405 (2010).

[15] I. Klich, Lower entropy bounds and particle number fluctuations in a Fermi sea, J. Phys. A 39, L85 (2006).

[16] F.N.C Paraan, J. Molina-Vilaplana, V. E. Korepin, and S. Bose, Entanglement in bipartite pure states of an interacting boson gas obtained by local projective measurements, 1105.1211.

[17] M. Haque, O. Zozulya, and K. Schoutens, Entanglement Entropy in Fermionic Laughlin States, Phys. Rev. Lett. 98, 060401 (2007); H. Li and F. D. M. Haldane, Entanglement Spectrum as a Generalization of Entanglement Entropy: Identification of Topological Order in Non-Abelian Fractional Quantum Hall Effect States, Phys. Rev. Lett. 101, 010504 (2008).

[18] M. Haque, O. Zozulya, K. Schoutens, Entanglement between particle partitions in itinerant many-particle states, J. Phys. A 42, 504012 (2009). 
[19] P. Calabrese, M. Minchev, and Ettore Vicari, The entanglement entropy of one-dimensional gases, Phys. Rev. Lett. 107, 020601 (2011).

[20] P. Calabrese, M. Campostrini, F. Essler, and B. Nienhuis, Parity effects in the scaling of block entanglement in gapless spin chains, Phys. Rev. Lett. 104, 095701 (2010)

[21] P. Calabrese and F. H. L. Essler, Universal corrections to scaling for block entanglement in spin-1/2 XX chains J. Stat. Mech. (2010) P08029.

[22] J. C. Xavier and F. C. Alcaraz, Renyi Entropy and Parity Oscillations of the Anisotropic Spin-s Heisenberg Chains in a Magnetic Field, Phys. Rev. B 83, 214425 (2011); M. Dalmonte, E. Ercolessi, and L. Taddia, Estimating Quasi-long-range Order via Renyi Entropies, 1105.3101.

[23] E.H. Lieb and W. Liniger, Exact Analysis of an Interacting Bose Gas. I. The General Solution and the Ground State, Phys. Rev. 130, 1605 (1963).

[24] L. Tonks, The Complete Equation of State of One, Two and Three-Dimensional Gases of Hard Elastic Spheres, Phys. Rev. 50, 955 (1936); M. Girardeau, ,"Relationship between Systems of Impenetrable Bosons and Fermions in One Dimension", J. Math. Phys. 1, 516 (1960); M. Girardeau, Permutation Symmetry of Many-Particle Wave Functions, Phys. Rev. 139, B500 (1965).

[25] V. Alba, L. Tagliacozzo, and P. Calabrese, Entanglement entropy of two disjoint blocks in critical Ising models, Phys. Rev. B 81 (2010) 060411; V. Alba, L. Tagliacozzo, and P. Calabrese, Entanglement entropy of two disjoint intervals in c=1 theories J. Stat. Mech. P06012 (2011).

[26] F Igloi and I Peschel, On reduced density matrices for disjoint subsystems, 2010 EPL 8940001.

[27] M Fagotti and P Calabrese, Entanglement entropy of two disjoint blocks in XY chains, J. Stat. Mech. (2010) P04016.

[28] B-Q Jin and V.E. Korepin, Quantum spin chain, Toeplitz determinants and Fisher-Hartwig conjecture, J. Stat. Phys. 116, 79 (2004).

[29] I Peschel, Calculation of reduced density matrices from correlation functions, J. Phys. A 36, L205 (2003); On the reduced density matrix for a chain of free electrons J. Stat. Mech. (2004) P06004.

[30] I. Peschel and V. Eisler, Reduced density matrices and entanglement entropy in free lattice models, J. Phys. A 42, 504003 (2009).

[31] S Furukawa, V Pasquier, and J Shiraishi, Mutual information and compactification radius in a c=1 critical phase in one dimension, Phys. Rev. Lett. 102, 170602 (2009).

[32] P Calabrese, J Cardy, and E Tonni, Entanglement entropy of two disjoint intervals in conformal field theory, J. Stat. Mech. P11001 (2009); P Calabrese, J Cardy, and E Tonni, Entanglement entropy of two disjoint intervals in conformal field theory II, J. Stat. Mech. P01021 (2011).

[33] M. E. Fisher and R. E. Hartwig, Toeplitz determinants: some applications, theorems, and conjectures, Adv. Chem. Phys. 15, 333 (1969).

[34] E. L. Basor and C. A. Tracy, The Fisher-Hartwig conjecture and generalizations, Physica A 177, 167 (1991); E. L. Basor and K. E. Morrison, The Fisher-Hartwig conjecture and Toeplitz eigenvalues, Linear Algebra and Its Applications 202, 129 (1994).

[35] J I Latorre and A Riera, A short review on entanglement in quantum spin systems, J. Phys. A 42, 504002 (2009).

[36] E. L. Basor and C. A. Tracy, The Fisher-Hartwig conjecture and generalizations, Physica A 177, 167 (1991).

[37] J. Cardy and P. Calabrese, Unusual Corrections to Scaling in Entanglement Entropy, J. Stat. Mech. P04023 (2010).

[38] P. Calabrese, J. Cardy, and I. Peschel, Corrections to scaling for block entanglement in massive spin chains, J. Stat. Mech. (2010) P09003; E Ercolessi, S Evangelisti, F Franchini, and F Ravanini, Essential singularity in the Renyi entanglement entropy of the one-dimensional XYZ spin-1/2 chain, Phys. Rev. B 83, 012402 (2011).

[39] M. Campostrini and E. Vicari, M Campostrini and E Vicari, Scaling of bipartite entanglement in one-dimensional lattice systems with a trapping potential, J. Stat. Mech. (2010) P08020; Quantum critical behavior and trap-size scaling of trapped bosons in a one-dimensional optical lattice, Phys. Rev. A 81, 063614 (2010).

[40] M Fagotti, P Calabrese, and J E Moore, Entanglement spectrum of random-singlet quantum critical points, Phys. Rev. B 83, 045110 (2011).

[41] J C Xavier, Entanglement entropy, conformal invariance and the critical behavior of the anisotropic spin-S Heisenberg chains: A DMRG study, Phys. Rev. B 81, 224404 (2010).

[42] P.J. Forrester and N.S. Witte, Discrete Painlevé equations, Orthogonal Polynomials on the Unit Circle and $N$-recurrences for averages over U(N) - PVI $\tau$-functions, arXiv:math-ph/0308036 P.J. Forrester and N.S. Witte, Bi-orthogonal Polynomials on the Unit Circle, regular semiclassical Weights and Integrable Systems, Constructive Approximation 24, 201 (2006). 
[43] M. Fagotti and P. Calabrese, Universal parity effects in the entanglement entropy of XX chains with open boundary conditions, J. Stat. Mech. P01017 (2011).

[44] F. C. Alcaraz and M. S. Sarandy, Phys. Rev. A 78, 032319 (2008).

[45] V Alba, M Fagotti, and P Calabrese, Entanglement entropy of excited states, J. Stat. Mech. (2009) P10020.

[46] F. C. Alcaraz, M. I. Berganza, and G. Sierra, Entanglement of low-energy excitations in Conformal Field Theory, Phys. Rev. Lett. 106, 201601 (2011).

[47] P. Deift, A. Its, and I. Krasovsky, Asymptotics of Toeplitz, Hankel, and Toeplitz+Hankel determinants with Fisher-Hartwig singularities, 0905.0443.

[48] P. Fendley, H. Saleur and N. Warner, Exact solution of a massless scalar field with a relevant boundary interaction, Nucl. Phys. B 430, 577 (1994).

[49] J. L. Cardy, O.A. Castro-Alvaredo, and B. Doyon, Form factors of branch-point twist fields in quantum integrable models and entanglement entropy, J. Stat. Phys. 130 (2008) 129.

[50] J. L. Cardy, Conformal Invariance and Surface Critical Behavior, 1984 Nucl. Phys. B 240514

[51] P. Calabrese and J. Cardy, Entanglement and correlation functions following a local quench: a conformal field theory approach, J. Stat. Mech. P10004 (2007); V. Eisler and I. Peschel, Evolution of entanglement after a local quench, J. Stat. Mech. P06005 (2007); V. Eisler, D. Karevski, T. Platini, I. Peschel, Entanglement evolution after connecting finite to infinite quantum chains, J. Stat. Mech. (2008) P01023; P. Calabrese, C. Hagendorf, P. Le Doussal Time evolution of $1 \mathrm{D}$ gapless models from a domain-wall initial state: SLE continued?, J. Stat. Mech. (2008) P07013; J.-M. Stphan and J. Dubail, Local quantum quenches in critical one-dimensional systems: entanglement, Loschmidt echo, and light-cone effects, 1105.4846; U. Divakaran, F. Igloi, H. Rieger, Non-equilibrium quantum dynamics after local quenches, 1105.5317.

[52] I. Klich and L. Levitov, Quantum noise as an entanglement meter, Phys. Rev. Lett. 102, 100502 (2009); B. Hsu, E. Grosfeld, and E. Fradkin, Quantum noise and entanglement generated by a local quantum quench, Phys. Rev. B 80, 235412 (2009); H. F. Song, C. Flindt, S. Rachel, I. Klich, K. Le Hur, Entanglement from Charge Statistics: Exact Relations for ManyBody Systems, Phys. Rev. B 83, 161408(R) (2011); J. Cardy, Measuring entanglement using quantum quenches, Phys. Rev. Lett. 106, 150404 (2011); 